\title{
Genome-Wide Mapping of Histone Modifications in Two Species of Leptosphaeria Maculans Showing Contrasting Genomic Organization and Host Specialization
}

\section{Jessica Louise Soyer ( $\boldsymbol{\nabla}$ jessica.soyer@inrae.fr )}

INRAE Île-de-France-Versailles-Grignon: Institut National de Recherche pour I'Agriculture I'Alimentation et I'Environnement Centre lle-de-France-Versailles-Grignon https://orcid.org/0000-0002-9424-1053

\section{Colin Clairet}

INRAE Île-de-France-Versailles-Grignon: Institut National de Recherche pour l'Agriculture I'Alimentation et I'Environnement Centre lle-de-France-Versailles-Grignon

\section{Elise J. Gay}

INRAE Île-de-France-Versailles-Grignon: Institut National de Recherche pour I'Agriculture l'Alimentation et I'Environnement Centre Ile-de-France-Versailles-Grignon

\section{Nicolas Lapalu}

INRAE Île-de-France-Versailles-Grignon: Institut National de Recherche pour I'Agriculture l'Alimentation et I'Environnement Centre lle-de-France-Versailles-Grignon

\section{Thierry Rouxel}

INRAE Île-de-France-Versailles-Grignon: Institut National de Recherche pour l'Agriculture l'Alimentation et I'Environnement Centre Ile-de-France-Versailles-Grignon

\section{Eva H. Stukenbrock}

Max Planck Institute for Evolutionary Biology: Max-Planck-Institut fur Evolutionsbiologie Isabelle Fudal

INRAE Île-de-France-Versailles-Grignon: Institut National de Recherche pour l'Agriculture l'Alimentation et I'Environnement Centre lle-de-France-Versailles-Grignon

\section{Research}

Keywords: Leptosphaeria maculans, ChIP-seq, RNA-seq, comparative epigenomics, chromatin, heterochromatin, effectors, host-pathogen interaction

Posted Date: September 18th, 2020

DOI: https://doi.org/10.21203/rs.3.rs-73550/v1 
License: (c) (i) This work is licensed under a Creative Commons Attribution 4.0 International License. Read Full License 
1 Genome-wide mapping of histone modifications in two species of Leptosphaeria maculans

2 showing contrasting genomic organization and host specialization

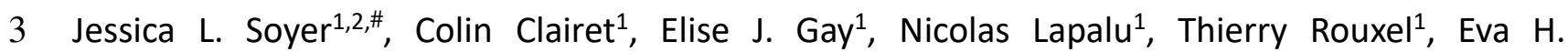

4 Stukenbrock $^{2, *}$, Isabelle Fudal ${ }^{1,} \neq$

5 1'Université Paris-Saclay, INRAE, AgroParisTech, UMR BIOGER, 78850, Thiverval-Grignon, France

$6 \quad{ }^{2}$ Max Planck Institute for Evolutionary Biology, August-Thienemann-Str. 2, 24306 Plön, and

7 Christian-Albrechts University of Kiel, Am Botanischen Garten 1-9, 24118 Kiel, Germany

$8 \quad{ }^{\ddagger}$ Co-last authors

$9 \quad{ }^{\#}$ Corresponding author: J.L. Soyer; jessica.soyer@inrae.fr

10

\section{Word count}

12 Abstract: 246; Introduction: 1080; Materials and Methods: 991; Results: 2319; Discussion: 1843

13

\section{Keywords}

15 Leptosphaeria maculans, ChIP-seq, RNA-seq, comparative epigenomics, chromatin, 16 heterochromatin, effectors, host-pathogen interaction. 
ABSTRACT

Background: In plant-associated fungi, the epigenome is increasingly recognized as an important regulator of the expression of genes involved in interaction with the host plant. Leptosphaeria

21 maculans 'brassicae' (Lmb) and Leptosphaeria maculans 'lepidii' (Lml) are closely-related

22 phytopathogenic species that exhibit a large macrosynteny but contrasting genome structure.

$23 \mathrm{Lmb}$ has more than $30 \%$ of repeats clustered in large repeat-rich regions, while the Lml genome

24 has only a small amount of evenly distributed repeats. Repeat-rich regions of Lmb are enriched

25 in effector genes, expressed during plant infection. The distinct genome structures of Lmb and

$26 \mathrm{Lml}$ provide an excellent model for comparing the organization of pathogenicity genes in relation

27 to the chromatin landscape in two closely related phytopathogenic fungi. Here, we performed 28 chromatin immunoprecipitation (ChIP) during axenic culture, targeting histone modifications 29 typical for heterochromatin or euchromatin, combined with transcriptomic analysis to analyse 30 the influence of chromatin organisation on gene expression.

31 Results: In both species, we found that facultative heterochromatin are enriched with genes

32 lacking functional annotation, including numerous effector and species-specific genes. Notably, 33 orthologous genes located in H3K27me3-domains are enriched with effector genes. Compared 34 to other fungal species, including $\mathrm{Lml}, \mathrm{Lmb}$ is distinct in having large H3K9me3-domains 35 associated with repeat-rich regions that contain numerous species-specific effector genes.

36 Conclusion: Discovery of these two distinctive heterochromatin landscapes now raises questions 37 about their involvement in the regulation of pathogenicity, the dynamics of these domains 38 during plant infection, and the selective advantage to the fungus to host effector genes in 39 H3K9me3or H3K27me3-domains. 


\section{Background}

42 Each year, hundreds of millions of tons of agricultural crops are devastated by plant pathogenic

43 fungi or made unfit for consumption due to contamination by mycotoxins (1). Management

44 strategies to control fungal infection mainly involve chemical control or breeding for naturally

45 resistant crop cultivars. However, fungal plant pathogens have proven capable of rapidly evolving

resistance against fungicides (1) and to overcome specific plant resistance genes within a few

years (for instance in Leptosphaeria maculans; 2), emphasising the need for improved control methods.

Understanding the determinants of the extreme adaptive abilities of fungal plant pathogens is a critical issue for the development of effective and sustainable control methods. In that respect, comparative and population genomic analyses have provided new insights into the evolutionary dynamics of fungal plant pathogens (e.g. 3-5). Notably, transposable elements (TE) have been shown to play a crucial role in shaping the genome structure of plant-pathogenic fungi. TEs are often organized in clusters, compartmentalizing the genome into gene-rich regions and TE-rich regions (e.g. in L. maculans and in Mycosphaerella fijiensis; 4, 6, 7). While the gene density in TErich regions is low, genes located in these regions have been shown to evolve faster than genes located in TE-poor regions $(8,9)$. Interestingly, rapidly evolving genes in TE-rich regions have, in many cases, been identified as genes involved in niche adaptation and notably effector genes. Effectors are considered as key elements of pathogenesis, allowing pathogens to circumvent host recognition, impede defence reactions and facilitate host invasion. Effectors are mainly secreted proteins, but can also correspond to secondary metabolites and small RNAs (10, 11, 
62 12). Plants have evolved strategies to recognize and counteract effectors, exposing them to a

63 strong selection pressure by the host immune system $(2,13,14)$. Indeed, in the course of the co-

64 evolution between a pathogen and its host, the host has developed an active immune system

65 allowing the direct or indirect recognition of some effector molecules, to activate defence

66 responses, often involving a local cell death, called the hypersensitive response. Effectors that

67 can be recognized by the host are called avirulence proteins $(10,15)$.

68 Leptosphaeria maculans 'brassicae' (hereinafter referred to as Lmb) belongs to the

69 Dothideomycete class of Ascomycete fungi and is responsible for causing stem canker of oilseed

70 rape (Brassica napus). Lmb displays a complex, hemibiotrophic life cycle, during which it

71 alternates between different nutritional modes on its host plant. It causes necrosis on different

72 plant organs: leaves, more rarely seedpods, and the base of the stem, causing lodging of the

73 plant and yield losses (16). The most efficient method of disease control relies on the use of

74 major resistance genes present in oilseed rape and other Brassica species. Although efficient,

75 this control method is not sustainable, as Lmb is able to "break down" novel sources of genetic

76 resistance rapidly (2). One third of the $\mathrm{Lmb}$ genome is made of TE-rich regions. These regions are

77 enriched with putative effector genes and include all currently known avirulence genes. These

78 avirulence genes are highly expressed in the first seven days of leaf and cotyledon infection (17-

79 26). Another set of putative proteinaceous effector genes are located in gene-rich regions of the

80 genome and are specifically expressed during stem infection (27).

81 In eukaryotic cells, chromatin can adopt different conformational states directly influencing gene

82 expression: gene-rich euchromatin, sheltering constitutively expressed genes, and gene-poor 83 heterochromatin, in which genes are silent. The different chromatin states are characterized by 
84 different post-translational modifications of histones around which DNA is wrapped. Typically, 85 heterochromatin is enriched in the trimethylation of the lysine 9 of histone $\mathrm{H} 3$ (H3K9me3) and 86 lysine 27 (H3K27me3) while euchromatin is enriched in the di- (or tri-) methylation of the lysine 874 of histone H3 (H3K4me2) (28). Recent research has focused on dynamic changes in DNA accessibility and how these may play fundamental roles in the adaptation of different individuals to abiotic and biotic stresses $(29,30)$. In fungal plant pathogens or endophytes, evidence is accumulating that transcriptional reprogramming of effector genes (either proteinaceous or

91 metabolic) is tightly controlled by chromatin-based regulatory mechanisms (for example in

92 Fusarium graminearum, Epichloe festucae, Lmb and Zymoseptoria tritici; 31-35). In Lmb, the

93 location of avirulence genes in TE-rich regions has an influence on their evolution under

94 selection pressure $(6,36)$ and plays a role in regulating their expression during axenic culture via 95 deposition of H3K9me3 (33).

96 Lmb belongs to the L. maculans / Leptosphaeria biglobosa species complex, comprising species 97 with different host specialization and genome organization (4). Within the species complex, the L. maculans species infecting oilseed rape, $L \mathrm{mb}$, is the only one having large regions of its genome enriched with TEs while other genomes have a low TE-content ( 3-15\% compared to more than $30 \%$ TEs in Lmb; 4, 6, 37). For example, the genome of the species that is most closely related to Lmb, Leptosphaeria maculans 'lepidii' (hereinafter referred to as $\mathrm{Lml}$ ), which infects

102 crucifers such as Lepidium sativum, and to a lesser extent Camelina sativa and Brassica rapa

103 (38), has a low TE content (3\% of TEs; 4). Interestingly, the genomes of Lmb and Lml show a high

104 level of macrosynteny, with only a few intra-chromosomal inversions, but differ in their TE 105 content with Lmb having undergone a massive TE expansion 5 million years ago corresponding 
106 to the speciation date (4). Invasion of TEs in the genome of Lmb has shaped its genome, with

107 alternating TE-rich regions and gene-rich regions. In contrast, the Lml genome shows a

108 homogeneous TE distribution along the chromosomes (4).

109 The distinct genome organisation shown by Lmb and $\mathrm{Lml}$ thus provides us with a model of

110 choice for comparing epigenomic organization in two closely related phytopathogenic fungi, to

111 determine the genomic location of pathogenicity/effector genes in relation to the chromatin

112 landscape and the influence of chromatin structure on gene expression. Comparative

113 epigenomic analyses, at the intra- or inter-species levels, are very sparse and this remains an

114 underexplored field of study, at least in fungi $(39,40)$. We present here the first comparative

115 epigenomic analysis of both euchromatin and heterochromatin marks in two closely related

116 phytopathogenic fungi. We first performed ChIP-seq and RNA-seq during axenic culture to

117 compare the distribution of three histone modifications, H3K4me2, H3K9me3 and H3K27me3,

118 and then investigated whether chromatin organization is conserved between Lmb and Lml by

119 assessing whether orthologous genes, and pathogenicity-related genes, are located in similar

120 chromatin domains in each species. Lastly, we assessed the influence of the chromatin landscape

121 on gene expression during axenic growth, focusing mostly on pathogenicity related genes that

122 may be species-specific.

123

\section{Methods}

125 Fungal isolates

126 The isolate v23.1.3 of L. maculans 'brassicae' and the isolate IBCN84 of L. maculans 'lepidii' were 127 used throughout the analyses (Rouxel et al., 2011; Grandaubert et al., 2014). Fungal cultures 
128 were maintained as described previously (41). For chromatin immunoprecipitation and

129 transcriptomic analyses performed during in vitro growth, mycelium of $\mathrm{Lmb}$ and $\mathrm{Lml}$ were grown

130 on V8 agar medium at $25^{\circ} \mathrm{C}$ for seven days. Then 10 plugs of mycelium were inoculated into 100

$131 \mathrm{ml}$ of Fries liquid medium in Roux bottle. Mycelia were harvested after growing for 7 days at

$13225^{\circ} \mathrm{C}$, filtered and washed thoroughly with distilled water, and immediately placed in liquid

133 nitrogen until further used. ChIP experiments were performed on fresh material.

135 Chromatin immunoprecipitation and high-throughput sequencing

136 ChIP was performed from freshly-harvested mycelium grown in Fries liquid culture, as described

137 in Soyer et al. (42), with minor modifications. ChIP was performed on native material (without

138 crosslinking) using antibodies targeting histone modifications H3K4me2 (Merck ref. 07-030),

139 H3K9me3 (Active Motif, Carlsbad, CA, USA; ref. 39161), or H3K27me3 (Active Motif, Carlsbad,

140 CA, USA; ref. 39155). Three different ChIPs (i.e. three biological replicates) were performed for

141 each of the histone modifications. Libraries were prepared from all biological replicates,

142 individually, according to the Illumina TruSeq protocol "Ultra Low Input DNA library". Libraries

143 were sequenced with an Illumina HiSeq 2000 genome analyzer at the Max Planck Genome

144 centre Cologne, Germany (https://mpgc.mpipz.mpg.de/home/). Sequencing data are available

145 under the GEO accession number GSE150127.

146

147 Analysis of ChIP-seq data and identification of significantly enriched domains

148 Analysis of ChIP-seq datasets was performed as described in Schotanus et al. (43). Quality of

149 Illumina $\quad$ was analysed using $\quad$ FastQC 
150 (https://www.bioinformatics.babraham.ac.uk/projects/fastqc/). Based on results of this analysis,

151 ten bp were trimmed from the $5^{\prime}$ end. Processed reads were mapped on the reference genome

152 of Lmb (37) or Lml (4) using Bowtie2 (44) with default parameters (Additional table 1). Peak

153 calling analysis was performed on each ChIP sequencing dataset, to identify significantly

154 enriched domains for either H3K4me2, H3K9me3 or H3K27me3, using RSEG (45). A domain was

155 considered if identified in at least two out of the three biological replicates. The Integrative

156 Genome Viewer (46) was used to visualize location of each domain along genomes of $\operatorname{Lmb}$ and

$157 \mathrm{Lml}$ according to other genome features (gene annotation, TE-annotation, GC-content).

158 Coverage of the histone modifications was assessed in $10 \mathrm{~kb}$ non-overlapping sliding windows

159 along the supercontigs and correlation analyses, using Kendall's $\mathcal{T}$ correlation coefficient, were

160 performed between biological replicates to check for reproducibility (Additional tables 2 and 3).

161 In order to assess significant enrichment of H3K4me2, H3K9me3 or H3K27me3 in certain

162 categories of genes (such as proteinaceous or metabolic effector-encoding genes), a Chi ${ }^{2}$ test

163 was applied to compare the expected proportion of a given category of genes across the entire

164 genome to the observed distribution of the gene category in H3K4me2-, H3K9me3- or

165 H3K27me3-domains (35). Enrichment was considered significant with a $P$ value $<0.01$; analyses

166 were done using R, version 3.0.2 (www.r-project.org).

167

168 RNA extraction, RNA-sequencing and expression analysis

169 Total RNA was extracted from mycelium grown for one week in Fries liquid medium as previously

170 described (18). The NEBNext Ultra Directional RNA Library Prep Kit for Illumina (cat. \# E7420L

171 New England BioLabs) was used to prepare RNA-seq libraries and sequencing was performed on 
172 a HiSeq 4000 Illumina genome analyser using 50 paired-end reads. Raw reads were then pre-

173 processed with Trimmomatic (47) to remove short reads (<30 bp) and eliminate sequencing

174 adaptors. Cleaned reads were then mapped against each genome using STAR with default

175 parameters (48; Additional table 1). Gene expression was evaluated using the rpkm_count

176 function of EdgeR (49). Genes with RPKM $\geq 2$ were considered expressed. Sequencing data are

177 available under the GEO accession number GSE150127.

178

179 Identification of orthologues and annotation of genes encoding proteinaceous effectors in Lmb

180 and Lml

181 The genome of $\operatorname{Lmb}$ encodes 13,047 proteins and that of $\operatorname{Lml} 11,272$ proteins $(4,37)$.

182 Orthologous proteins between $\mathrm{Lmb}$ and $\mathrm{Lml}$ were identified using OrthoFinder with default

183 parameters (50).

184 Based on the new assembly and annotation available for the genome of Lmb (37), an updated 185 repertoire of putative proteinaceous effector genes (encoding Small Secreted Proteins, SSP) has

186 been predicted. Therefore, to compare effector repertoires of $\mathrm{Lmb}$ and $\mathrm{Lml}$, the same pipeline

187 for the prediction of putative effector genes was applied to both species. Signal peptide and 188 subcellular localization were predicted by SignalP version 4.1 (51) and TargetP version 1.1 (52)

189 respectively. Transmembrane domains were predicted by TMHMM version 2.0. The predicted

190 secretome contained all proteins with no more than one transmembrane domain and either a

191 predicted signal peptide or a predicted extracellular localization. The final effector repertoires

192 were created by applying a size cut-off of 300 amino acids on the predicted secretome. In

193 parallel, EffectorP version 1.0 (53) was used on the predicted secretome and, for Lml, four 
194 proteins were predicted as effectors with a size higher than 300 amino acids. These four proteins

195 were added to the SSP set. This predicted a repertoire of 1,080 and 892 SSP-encoding genes for

$196 \mathrm{Lmb}$ and for Lml, respectively.

197

198 Analysis of GO enrichment

199 Gene Ontology (GO) annotations of the $\mathrm{Lmb}$ and $\mathrm{Lml}$ genes were retrieved from Dutreux et al. 200 (37) and Grandaubert et al. (4) respectively. GO term enrichment analysis of the H3K4me2-, 201 H3K9me3- and H3K27me3-associated genes was performed with the plug-in Biological networks 202 Gene ontology (BinGo; v3.0.3) of the cytoscape software (54). List of genes submitted to BINGO 203 were considered as significantly enriched for a given GO term with an associated False Discovery 204 Rate $(F D R) \leq 0.01$ for the biological processes.

$205 \mathrm{~A} \mathrm{Chi}^{2}$ test, or a Fisher's exact test for small sized-population, were applied to identify significant 206 enrichment of H3K4me2-, H3K9me3- or H3K27me3-domains for certain categories of genes: the 207 expected proportion of a given category of genes across the entire genomes of Lmb or Lml was 208 compared to the observed distribution of the gene category in the H3K4me2-, H3K9me3- or 209 H3K27me3-domains. Enrichment was considered significant with a $P$ value $<0.01$. All analyses 210 were done using R, version 3.0.2 (www.r-project.org).

\section{Results}

213 A different genome organisation but similar epigenomic properties in both genomes

214 To assess the genome-wide distribution of histone marks in $\mathrm{Lmb}$ and $\mathrm{Lml}$ during axenic growth, 215 we performed ChIP-seq experiments using antibodies against histone modifications H3K4me2, 
216 H3K9me3 and H3K27me3 (Additional table 1). Mapping of the ChIP-seq data was followed by 217 identification of significantly enriched domains, for any of the histone modifications targeted, 218 and reproducibility of the biological replicates was assessed (Additional tables 2 and 3). Based on 219 the genomic coordinates of the H3K4me2-, H3K9me3- and H3K27me3-domains, the number of 220 bases associated with any of the three histone modifications was evaluated, for each genome, in $22110 \mathrm{~kb}$ sliding-windows. In the genomes of $\mathrm{Lmb}$ and $\mathrm{Lml}$, the proportion of $\mathrm{H} 3 \mathrm{KK} 4 \mathrm{me} 2$ and 222 H3K27me3 was similar (H3K4me2: 39\% in Lmb vs. 32\% in Lml; H3K27me3: 19\% in Lmb vs. $13 \%$ in $223 \mathrm{Lml}$ ) while the proportion of $\mathrm{H} 3 \mathrm{~K} 9 \mathrm{me} 3$ was strikingly different ( $33 \%$ and $4 \%$ for $\mathrm{Lmb}$ and $\mathrm{Lml}$, 224 respectively) (Additional tables 4 and 5). While the median size of the H3K4me2- and 225 H3K27me3-domains was similar in both genomes, the Lmb genome displayed extremely large 226 H3K9me3-domains, with a domain encompassing up to $230 \mathrm{~kb}$, while the maximum size of the 227 H3K9me3-domains in Lml was only $12 \mathrm{~kb}$ (Figures $1 ; 2$ ).

228 In the genomes of $\mathrm{Lmb}$ and $\mathrm{Lml}, \mathrm{H} 3 \mathrm{~K} 4 \mathrm{me} 2$ - and H3K9me3-domains were mutually exclusive 229 (Kendall's $T$ : -0.57 and $-0.16, P<2.2 .10^{-16}$ for $\mathrm{Lmb}$ and $\mathrm{Lml}$ respectively; Figure 2; Additional 230 figure 1; Tables 1 and 2). We identified a positive correlation between the location of TEs and 231 H3K9me3-domains (Kendall's $T: 0.87$ and $0.68, P<2.2 .10^{-16}$ for $\mathrm{Lmb}$ and $\mathrm{Lml}$ respectively; Figure 232 2; Figure 3A, 3C; Figure S1; Tables 1 and 2) and between the location of coding sequences (CDS) 233 and H3K4me2 (Kendall's $T: 0.55$ and $0.25, P<2.2 .10^{-16}$ for $\mathrm{Lmb}$ and $\mathrm{Lml}$ respectively; Figure 2;

234 Figure S1; Figure 3A, 3C; Tables 1 and 2). In the genome of Lmb, a weak correlation was 235 demonstrated between CDS and H3K27me3-domains (Kendall's T: 0.12, $P<2.2 .10^{-16}$; Figure S1; 236 Tables 1, 2). For $\mathrm{Lmb}$ and $\mathrm{Lml}$, the coverage of H3K9me3 and H3K27me3 was not homogeneous 237 across all SCs because some are $>2$-fold more highly enriched with these histone modifications 
238 compared to others. An extreme example of this situation was identified on the dispensable

239 chromosome of $\operatorname{Lmb}(6,55)$, which is extremely enriched in TEs compared to the rest of the

240 genome (35\% of TEs in the core genome and $93 \%$ of TEs in the dispensable chromosome).

241 Consistently, a strong enrichment in H3K9me3 was observed in the dispensable chromosome

242 (32\% in the core genome and $90 \%$ in the dispensable chromosome), with only a few H3K4me2-

243 and H3K27me3-domains (Figure 3A and 3B). In Lmb, we found that many sub-telomeric regions

244 showed overlaps between H3K9me3 and H3K27me3, whereas these marks did not overlap on

245 the chromosome arms, even within large TE-rich regions (Figure 2). The assembly quality of the

$246 \mathrm{Lml}$ genome was not sufficient to allow us to evaluate whether this was a common feature

247 between the two species.

Table 1. Correlation between transposable elements, coding sequences and histone modifications in the L. maculans 'brassicae' genome.

\begin{tabular}{cccccc}
\hline & CDS & TE & H3K4me2 & H3K9me3 & H3K27me3 \\
CDS & 1 & -0.63 & 0.55 & -0.60 & 0.12 \\
TE & -0.63 & 1 & -0.58 & 0.87 & -0.25 \\
H3K4me2 & 0.55 & -0.58 & 1 & -0.57 & -0.11 \\
H3K9me3 & -0.60 & 0.87 & -0.57 & 1 & -0.24 \\
H3K27me3 & 0.12 & -0.25 & -0.11 & -0.24 & 1 \\
\hline The coverage of transposable elements (TE), coding sequences (CDS) and histone \\
modifications (H3K9me3, H3K27me3, H3K4me2) along the genome of L. maculans \\
'brassicae' was analysed in 1000 bp sliding windows. A Kendall's $T$ correlation \\
analysis was done using R.
\end{tabular}


Table 2. Correlation between transposable elements, coding sequences and histone modifications in the L. maculans 'lepidii' genome.

\begin{tabular}{cccccc}
\hline & CDS & TE & H3K4me2 & H3K9me3 & H3K27me3 \\
CDS & 1.00 & -0.22 & 0.25 & -0.25 & -0.05 \\
TE & -0.22 & 1.00 & -0.16 & 0.68 & 0.00 \\
H3K4me2 & 0.25 & -0.16 & 1.00 & -0.16 & -0.23 \\
H3K9me3 & -0.25 & 0.68 & -0.16 & 1.00 & 0.01 \\
H3K27me3 & -0.05 & 0.00 & -0.23 & 0.01 & 1.00 \\
\hline The coverage of transposable elements (TE), coding sequences (CDS) and histone \\
modifications (H3K9me3, H3K27me3, H3K4me2) along the genome of L. maculans \\
'lepidii' was analysed in 1000 bp sliding windows. A Kendall's T correlation analysis \\
was done using R.
\end{tabular}

250 H3K4me2-domains associate with genes involved in primary metabolism while H3K27me3-

251 domains shelter genes involved in niche adaptation and cell wall degradation

252 Genes associated with H3K4me2-, H3K9me3- or H3K27me3-domains were identified in the 253 genomes of $\mathrm{Lmb}$ and $\mathrm{Lml}$ during axenic growth. Overall, the number of genes associated with 254 any of the histone post-translational modifications was similar in both species, with more than $25550 \%$ of the predicted genes associated with H3K4me2, 14\% of the genes associated with 256 H3K27me3 and only a few genes associated with H3K9me3 (104 and 70 respectively in Lmb and 257 Lml; Figure 4). In Lmb, there was a higher number of genes associated with both H3K4me2 and $258 \mathrm{H} 3 K 27 m e 3$ than in $\mathrm{Lml}$ (2,044 and 361 for $\mathrm{Lmb}$ and $\mathrm{Lml}$, respectively) (Figure 4). In both 259 genomes, a GO annotation could be assigned to $30-40 \%$ of the predicted genes $(5,076$ and 3,725 260 genes for Lmb and Lml, respectively; 4, 37). Euchromatin was enriched with genes with a GO 261 annotation ( $\mathrm{X}^{2}$ test, $P<2.2 .10^{-16}$ ) because more than $40 \%$ of the genes associated with $\mathrm{H} 3 \mathrm{~K} 4 \mathrm{me} 2$ 262 had a GO annotation (3,276 and 2,581 genes for Lmb and Lml respectively). Between 20 and 
$26328 \%$ of the genes associated with H3K27me3 (572 and 288 genes for Lmb and Lml, respectively) 264 had a GO annotation. However, none of the genes located in H3K9me3-domains, H3K9/K27me3265 domains or H3K4me2/H3K27me3-domains had a GO annotation. In other words, genes located 266 in heterochromatin were enriched with genes lacking any functional annotation ( $X^{2}$ test, $P<$ 267 2.2.10-16). For both species, genes associated with H3K4me2 displayed a wide variety of 268 annotated functions corresponding to primary metabolism and basic cellular functions, such as 269 translation (GO:0006412; 206 genes) or cellular protein metabolic process (Additional tables 6, 270 7). For both species, only a few GO terms were identified among genes associated with 271 H3K27me3. Nevertheless, in Lmb these genes were significantly enriched in GO terms associated 272 with carbohydrate metabolic process (GO:0005975; 82 genes), oxydo-reduction process 273 (GO:0055114; 149 genes) and transmembrane transport (GO:0055085; 104 genes) $(P \leq 0.01)$ 274 (Additional table 8). As for $\mathrm{Lml}$, probably due to the lower number of genes associated to 275 H3K27me3, only a few GO annotation enrichments were detected. Only one GO enrichment was 276 found in common with Lmb, namely carbohydrate metabolic process (GO:0005975; 41 genes). 277 Other enrichments corresponded to a few genes classified as response to chemical (GO: 42221; 27820 genes), response to nitrogen compound (GO:1901698; 10 genes), response to 279 organophosphorus (GO: 46683, 10 genes) and others (Additional table 9). Although most GO 280 enrichments found among sets of genes associated with H3K27me3 in Lmb and Lml did not 281 overlap, both types of enrichments suggest that H3K27me3-associated genes might be involved 282 in stress response mechanisms, but may also be involved in feeding or cell wall degradation 283 processes during plant infection. 

domains are associated with silent genes during axenic growth

287 We performed a genome-wide transcriptomic analysis of fungal cultures grown in vitro, and we 288 correlated gene expression patterns with the distribution of histone modifications. In Lmb, 289 10,934 genes ( $83 \%$ of the predicted genes) and 7,735 genes in $\mathrm{Lml}$ (69\% of the predicted genes) were expressed during vegetative growth. Considering the top 100 most expressed genes of Lmb, 68 were associated with H3K4me2 while two were located within a H3K27me3-domain and 292 none in a H3K9me3-domain (data not shown). In Lml, 71 were located within a H3K4me2293 domain while five were located in a H3K27me3-domain (data not shown). These data were 294 confirmed at a genome-wide scale because H3K4me2-domains were enriched with genes 295 expressed during axenic culture $(7,104$ of the genes associated with H3K4me2 were expressed in $296 \mathrm{Lmb}$ and 5,338 in Lml; Figure 5; $P<2.2 .10^{-16}$ ). On the contrary, H3K9me3- and H3K27me3297 domains were enriched with genes that were silent during in vitro growth (Figure $5 ; P<2.2 .10^{-}$ $298{ }^{16}$ ). On the other hand, in both species, genes that were associated with both H3K4me2 and 299 H3K27me3 were expressed during axenic growth $(90 \%$ and $87 \%$ of the genes, respectively, in $300 \mathrm{Lmb}$ and $\mathrm{Lml}$ ) and their level of expression was similar to that of genes located in H3K4me2301 domains (Figure 5).

302 In both species, this transcriptomic analysis confirmed that genes located in a H3K4me2-domain 303 were more likely to be expressed while those located in H3K9me3- and H3K27me3-domains 304 were more likely to be silent. 
308 A total of 7,393 genes were conserved between both species. H3K4me2-domains were enriched

309 with genes conserved between these two species, for example 4,892 Lmb genes located in 310 euchromatin regions during growth in vitro were conserved $\left(X^{2}\right.$ test, $\left.P<2.2 .10^{-16}\right)$ and the vast 311 majority of these (4,298, i.e. $88 \%)$ were expressed during axenic growth in both $\mathrm{Lmb}$ and $\mathrm{Lml}$.

312 Most of the conserved genes associated with H3K4me2 were involved in primary metabolism as 313 mentioned above. Genes associated with both H3K4me2 and H3K27me3 were also enriched 314 with conserved genes (1,318 and 262 genes, respectively, in Lmb and Lml) representing more 315 than $65 \%$ of the genes in these domains $\left(X^{2}\right.$ test, $\left.P<5.2 .10^{-3}\right)$. Two genes involved in 316 heterochromatin assembly and maintenance were analysed previously through gene silencing 317 (LmHP1 and LmKMT1/DIM5; 33). LmHP1 is conserved in Lml and located in euchromatin in both 318 species. LmKMT1 is also conserved in both species, being located in a H3K4me2/H3K27me3 319 domain in Lmb and within a H3K4me2/H3K9me3 domain in the case of Lml, during axenic 320 growth. In contrast, H3K27me3- and H3K9me3-domains were significantly enriched with 321 species-specific genes (a total of 865 genes and 661 genes, respectively, in Lmb and Lml; $\mathrm{X}^{2}$ test, $322 P<2.2 .10^{-16}$ ). Among genes located in heterochromatin in both species (either H3K9me3-, 323 H3K27me3- or H3K9/K27me3-domains), 445 were conserved between Lmb and Lml; of which 83 324 were expressed and 182 were repressed during axenic growth in both species. No GO 325 enrichment was found for these genes and predicted functions were sparse, with less than $45 \%$ 326 of them having a functional annotation. Strikingly, the main enrichment was in genes encoding 327 putative effectors (16\% of the genes). 
329 Heterochromatin domains are enriched with proteinaceous and metabolic effector genes

330 We then focused on candidate proteinaceous or metabolic effectors and wondered whether

331 they were conserved and showed a distinct pattern of histone modifications. In the genome of

332 Lmb, 2,478 genes (i.e. $12 \%$ of the total genes) were associated with TE-rich regions (i.e. located

333 within $2 \mathrm{~kb}$ distance of a TE sequence), of which 289 genes encoded putative proteinaceous

334 effectors. Hence, although a new prediction of the effector repertoire was performed here,

335 based on the new assembly of the Lmb genome, TE-rich regions were significantly enriched with

336 proteinaceous effector genes, as was already shown by Rouxel et al. (6; $X^{2}$ test, $\left.P=9.6 .10^{-16}\right)$.

337 Similarly, both H3K9me3- and H3K27me3-associated genes were significantly enriched with

338 putative proteinaceous effectors, as they represented, respectively, $36 \%$ and $14 \%$ of the genes

339 associated with these histone modifications in vitro ( $X^{2}$ test, $P<2.2 .10^{-16}$; Table 3 ). Likewise, in

340 Lml, TE-rich regions, H3K9me3- and H3K27me3-domains were significantly enriched with

341 putative proteinaceous effector genes while H3K4me2-domains were significantly depleted in

342 such genes compared to the rest of the genome (Table 3). Among the 1,080 putative

343 proteinaceous effector genes predicted in $\operatorname{Lmb}(8.2 \%$ of the total predicted genes) and the 892

344 putative effector genes of $\operatorname{Lml}$ (7.9\% of the total predicted genes), 274 were conserved between

345 both species. Hence, more than two-thirds of the effector repertoire is species-specific ( $X^{2}$ test, $P$

$\left.346<2.2 .10^{-16}\right)$, confirming our previous findings (4). Overall, orthologous effector-encoding genes

347 were associated with the same types of chromatin domains in Lmb and Lml. For example, $98 \%$ of

348 the Lmb effector genes located in a euchromatin environment were also associated with

$349 \mathrm{H} 3 \mathrm{~K} 4 \mathrm{me} 2 \mathrm{in} \mathrm{Lml}$ (82\% of $\mathrm{Lml}$ effector genes located in euchromatin regions were also associated

350 with H3K4me2 in Lmb). H3K27me3- and H3K9me3-domains were enriched with species-specific 
351 effector genes in both species (Table 3). As a striking example of the non-random location of

352 effector genes in the two genomes, and the enrichment of heterochromatin regions with

353 species-specific effectors, all nine currently known avirulence genes of Lmb were located in

354 H3K9me3-domains in vitro, consistent with the fact that these genes are located in TE-rich

355 genomic compartments; none of the nine was conserved in the genome of Lml. Twenty-eight

356 other proteinaceous effector-encoding genes were located in TE-rich, H3K9me3-domains in Lmb,

357 and only one of them had a putative ortholog in Lml. The 274 orthologous genes encoding

358 effectors were then investigated for their distribution in euchromatic/heterochromatic regions

359 during vegetative growth and for conservation of their location between orthologs. There was

360 no obvious bias in the distribution of chromatin marks among the 274 genes, which is

361 comparable to the overall distribution of marks among all genes of the effector repertoires (data

362 not shown). At the individual gene level, 86 of the 274 orthologs were located in a similar

363 chromatin domain in $\mathrm{Lmb}$ and $\mathrm{Lml}$, including 83 genes associated with $\mathrm{H} 3 \mathrm{~K} 4 \mathrm{me} 2$ and 47 genes

364 associated with H3K27me3 in both genomes. In contrast to avirulence genes, 'late' putative

365 effector-genes of Lmb (i.e. expressed during stem colonization) were located outside of TE-rich

366 regions (27). Three of the 11 experimentally validated 'late' effector genes were located in a

367 H3K4me2-domain while others were located in a H3K9me3- (two cases), a H3K27me3- (three

368 cases), a H3K9/K27me3- (one case) or a H3K4me2/K27me3-domain (two cases), suggesting that

369 'late' effector genes were also enriched with heterochromatin domains during growth in vitro.

370 Interestingly, and contrary to the trend observed for Lmb avirulence effector genes (and other

371 effector candidates associated with TE-rich regions), only two of the 11 'late' putative effector

372 genes, either located in H3K9me3- or H3K27me3-domains, were not conserved in Lml. Taken 
373 together, these data show that, independently of the species or the stage at which they are 374 expressed during infection, proteinaceous effector genes are enriched in H3K9me3- or 375 H3K27me3-domains during axenic growth.

376 The genomes of $\mathrm{Lmb}$ and $\mathrm{Lml}$ contain secondary metabolite gene clusters including key genes 377 encoding PKS (Polyketide Synthases) and NRPS (Non-Ribosomal Peptide Synthetases). Twenty378 seven such genes were predicted in $\mathrm{Lmb}$ among which 24 were conserved in $\mathrm{Lml}$, but they were 379 overall absent from other closely related species (4, 6; Additional table 10). Of these, only three 380 have been experimentally demonstrated to be involved in Lmb pathogenicity, namely the PKS 381 responsible for synthesis of abscisic acid (ABA), only expressed during cotyledon infection (56), 382 the PKS responsible for producing phomenoic acid (57), and the NRPS responsible for 383 synthetising sirodesmin, a toxin produced during stem infection $(58,59)$. The ABA PKS and all 384 seven genes of the cluster, which are intermingled with three TE-rich regions, were entirely 385 absent from the genome of $\mathrm{Lml}$, while the other two were conserved (Additional table 10). In $386 \mathrm{Lmb}, 81 \%$ of the PKS/NRPS-encoding genes were associated to H3K27me3- or 387 H3K4me2/H3K27me3-domains (22 PKS/NRPS; Fisher's exact test, $P=1.3 \cdot 10^{-7}$ ). Likewise in the Lml 388 genome, H3K27me3-domains were enriched with PKS/NRPS-encoding genes (12 PKS/NRPS; 389 Fisher's exact test, $P=1.7 .10^{-4}$ ), and all of them had orthologs associated with similar marks in 390 Lmb. The similar chromatin context also resulted in similar regulation in most of the cases, with 39119 of the orthologs being similarly expressed during in vitro growth (17 expressed and two 392 repressed; Additional table 10). 
395 The sister species L. maculans 'brassicae' and L. maculans 'lepidii' exhibit marked differences in

396 their genome organisation. Notably, Lmb has large TE-rich domains structuring the genome into

397 alternating gene-rich and TE-rich regions (6). In the genome of Lml, in contrast, TEs are evenly

398 distributed across the genome and no compartmentalisation of the genome is evident in

399 relation to TE location (4). The massive invasion of the Lmb genome by TEs occurred ca. 5 MYA

400 and was postulated to have been instrumental in the separation of the two species (4). This

401 invasion may also have contributed to the rise of $L m b$ as a successful pathogen of $B$. napus due

402 to the specific localisation of a number of candidate effector genes in TE-rich regions of the

403 genome $(4,6)$. Differences in genomic organization between these two closely-related species

404 could impact the underlying epigenomic landscape and have important consequences for fungal

405 biology and pathogenicity. This could provide distinct strategies to regulate the expression of

406 genes involved in stress response or pathogenicity, as it was shown in Lmb that histone

407 modification H3K9me3 is involved in the repression of avirulence genes during axenic growth

408 (33). To investigate this question, we here compared the genome-wide location of three

409 different histone modifications that are typically associated with euchromatin or

410 heterochromatin in these two closely-related phytopathogenic fungi. We found that differences

411 in the epigenomic landscape of $\mathrm{Lmb}$ and $\mathrm{Lml}$ are in accordance with their genome organization.

412 In Lmb, very large H3K9me3-domains are present, spanning large TE-rich regions, while such

413 extremely large H3K9me3-domains are not observed in the $\mathrm{Lml}$ genome. Our findings

414 corroborate previous epigenetic analyses of a few genes performed in vitro, pointing out that

415 avirulence genes are located in heterochromatin, but also demonstrate that putative effector

416 genes, independently of their expression pattern or their location in TE-rich or gene-rich regions, 
417 are enriched with heterochromatin during axenic culture.

418

419 Although $\mathrm{Lmb}$ and $\mathrm{Lml}$ have distinct genome organisations, they share a common distribution of 420 histone modifications throughout their genome during axenic culture. Gene-rich regions are 421 enriched with H3K4me2 and H3K27me3, while TE-rich regions are associated with H3K9me3. 422 The proportion of H3K9me3 in a genome often reflects the TE content, as was described in $Z$. 423 tritici (43). In Lmb, having more than $30 \%$ of $\mathrm{TE}, 33 \%$ of the genome is associated with $\mathrm{H} 3 \mathrm{~K} 9 \mathrm{me} 3$ 424 while the genome of $\mathrm{Lml}$, having a low TE content, shows a low enrichment in H3K9me3 (4\% of $425 \mathrm{H} 3 \mathrm{~K} 9 \mathrm{me3}$ ). Domains enriched with H3K4me2 and domains enriched with H3K9me3 are mutually 426 exclusive in the genomes of $\mathrm{Lmb}$ and $\mathrm{Lml}$, as was shown in Neurospora crassa, Fusarium 427 fujikuroi, and Z. tritici $(43,60,61,62)$. H3K27me3 has been detected in most filamentous fungi 428 investigated so far, except in Mucor, Rhizopus or Aspergilli such as Aspergillus nidulans (63; 429 reviewed in 64). In sub-telomeric regions of Lmb, H3K9me3 and H3K27me3-domains overlap 430 over repetitive sequences, which is also the case in $N$. crassa or $Z$. tritici $(43,60,61,65)$. In the 431 genome of $\mathrm{Lmb}$, TE-rich regions are enriched with H3K9me3 but, except for the sub-telomeric 432 regions, no enrichment in H3K27me3 was associated with TEs on chromosomal arms. This 433 contrasts with the organization of TE-rich regions in Z. tritici, which are enriched with both 434 heterochromatin modifications (43). The situation in Lmb is also different from that of Fusarium 435 oxysporum in which most TE sequences are embedded in H3K27me3-domains (66). We 436 confirmed that, as in most Eukaryotes, H3K4me2-domains are associated with expressed genes 437 while H3K9me3- and H3K27me3-domains are associated with silent genes. In Lmb, although the 438 locations of H3K4me2- and H3K27me3-domains do not overlap at a genome-wide scale, more 
439 than 2,000 genes were found to be associated with both histone methylations. This is strikingly 440 different to $\mathrm{Lml}$, where only 300 genes were associated with both histone modifications, and $Z$.

441 tritici, where 400 genes are embedded in such domains $(35,43)$. Bivalent domains are defined as

442 chromatin regions associated with both repressive and permissive histone modifications (67).

443 The large number of genes associated with H3K27me3 and H3K4me2 during axenic culture in 444 Lmb might indicate a biological specificity of this species and the existence of bivalent domains, 445 because these two modifications have antagonistic effects on gene expression (68). Altogether, 446 our findings show that, despite differences in genomic organization between $\mathrm{Lmb}$ and $\mathrm{Lml}$ (and 447 the other fungi in which epigenomic analyses were so far performed), the epigenomic landscape 448 is overall conserved.

450 While H3K9me3 and H3K27me3 are signatures of heterochromatin, H3K9me3 is considered to 451 be typical of constitutive heterochromatin, being associated with repeats and involved in 452 genome stability, whereas $\mathrm{H} 3 \mathrm{~K} 27 \mathrm{me} 3$ is considered to be associated with facultative 453 heterochromatin that is easily reversed to a euchromatin state under certain abiotic and biotic 454 stress conditions (69). However, dogmas regarding conventional definitions of facultative or 455 constitutive heterochromatin seem to be challenged in fungi, or at least in the few plant 456 pathogenic fungi in which epigenomic analyses have been performed. While the co-localization 457 of H3K9me3 and TEs in the $\mathrm{Lmb}$ and $\mathrm{Lml}$ genomes is consistent with a constitutive 458 heterochromatin state, the fact that H3K9me3-domains encompass genes that are expressed 459 during interaction with oilseed rape suggests that these regions may correspond to a category of 460 facultative heterochromatin in this species. Genes associated with H3K9me3 are almost all 
461 located in the middle of repeated elements, in sub-telomeric areas, or very close to the edge of 462 regions enriched with repeated elements. Moreover, some of these genes, including the nine 463 cloned avirulence genes of Lmb which are located within AT-rich isochores (e.g., AvrLm1 or 464 AvLm6; 17, 18) including subtelomeric regions (e.g. AvrLm3 or AvrLm10; 24, 26), are highly 465 transcribed upon host infection (6). This finding, together with other studies, questions the 466 "constitutive" nature of heterochromatin associated with H3K9me3. In contrast, the location of $467 \mathrm{H} 3 \mathrm{~K} 27 \mathrm{me} 3$ in the genomes of $\mathrm{Lmb}$ and $\mathrm{Lml}$ supports its association with facultative 468 heterochromatin. Indeed, we found H3K27me3 associated with coding sequences and enriched 469 with genes encoding proteinaceous and metabolic effectors or proteins involved in stress 470 responses. Most of these genes are silenced during vegetative growth but induced during plant 471 infection. In fungi, recent analyses also highlighted a role for H3K27me3 in genome organization 472 and stability. For instance, the dispensable chromosomes of $Z$. tritici are twice as rich in TEs as 473 the core chromosomes, and whereas there is no significant enrichment in H3K9me3 on the 474 dispensable chromosomes, they are entirely covered by H3K27me3 (43). In Z. tritici, the loss of 475 H3K27me3 was found to increase the stability of some accessory chromosomes (70). In Z. tritici 476 and $N$. crassa, H3K27me3 is relocated towards normal constitutive heterochromatin (i.e. 477 H3K9me3-domains) under genotoxic stress, such as the loss of H3K9me3 after inactivation of $478 K M T 1(70,71)$. Altogether, these findings suggest that although H3K27me3 is an important 479 regulator of gene expression involved in development or response to various stresses, it also 480 plays a role in the maintenance of genome integrity. In Lmb and Lml, no particular association of 481 H3K27me3 with TEs was identified and the dispensable chromosome of Lmb is not enriched 482 with H3K27me3. The only association of H3K27me3 with constitutive chromatin was at the 
483 chromosome ends of Lmb, where we found overlaps between H3K9me3 and H3K27me3. 484 Inactivation of KMT1 and KMT6 would help investigate whether H3K27me3 could also be 485 involved in genome stability and relocation of heterochromatin marks in Lmb and Lml.

487 The heterochromatin domains of $\mathrm{Lmb}$ and $\mathrm{Lml}$ are rich in species-specific genes, genes involved 488 in stress responses and putative proteinaceous or metabolic effectors. This supports the view 489 that chromatin remodelling mechanisms are an efficient way to rapidly modulate gene 490 expression under stress conditions or during biotic interactions, although the dynamics of 491 chromatin structure might not be the sole regulator of these complex biological processes (34, 492 72). This pattern is conserved in other plant-associated fungi, independently of their mode of 493 interaction with their host $(6,32,35,43,73,74,75,76,77)$. Even outside of TE-rich regions, 494 heterochromatin is often found enriched with pathogenicity-related genes, whether they 495 encode proteinaceous effectors or are involved in the production of secondary metabolites (e.g. $49631,32,35,43,62,66)$. Importantly, sets of genes up-regulated during host infection are found 497 associated with heterochromatin in vitro $(35,66,78)$. One of our initial postulates was that 498 invasion of the L. maculans genome by TEs contributed to the rise of Lmb as a pathogen 499 specialized on Brassicas with greater pathogenic abilities than the non-invaded sister species. 500 While $\mathrm{Lml}$ has never been found to be able to infect $B$. napus under our experimental conditions 501 or isolated from our experimental fields (M-H. Balesdent \& T. Rouxel, unpublished data), 502 previous work reported its ability to infect other crucifers (Petrie, 1969). Its isolation from 503 ascocarps on stems of Lepidium sp. also suggests its infection strategy is similar to that of Lmb 504 on B. napus. So far, all avirulence genes of $\mathrm{Lmb}$, which can be recognized by the plant immune 
505 system to set up defence reactions, are located in TE-rich regions and associated with H3K9me3.

506 In contrast, in both species we found conserved putative effector genes (either proteinaceous or 507 metabolic) harbored in similar H3K27me3 heterochromatin environments. Moreover, 'late' 508 effector genes are more conserved than avirulence genes between $\mathrm{Lmb}$ and $\mathrm{Lml}$, and usually do 509 not show presence/absence polymorphisms in Lmb (27). The contrasting heterochromatic 510 environment (H3K9me3 vs. H3K27me3) for avirulence genes and 'late' effector genes reflects a

511 very different adaptive behaviour because effector genes that are expressed early, and likely to 512 be "recognised" by the plant surveillance machinery at the onset of penetration, are also those 513 which are subject to accelerated evolution under selection (6). This suggests that there may be a

514 selective advantage for the fungus to partition genes more likely to be recognised by the host 515 plant within H3K9me3-domains. Nevertheless, the location of putative pathogenicity genes, 516 including orthologues, in similar heterochromatin regions in the genomes of $\mathrm{Lmb}$ and $\mathrm{Lml}$ 517 suggests that basic pathogenicity programs are independent of genome invasion by TEs, and 518 points to the likewise importance of chromatin context on transcriptome shaping during 519 infection. Taken together, these data support our previous hypothesis that the localization of 520 effector genes in plastic genomic compartments is an efficient way to regulate the expression of 521 sets of genes scattered throughout the genome that are involved in similar biological processes 522 (34).

\section{Conclusions}

525 To the best of our knowledge, previous comparative analyses of histone modifications have been 526 performed in model organisms such as mouse (79) but none considered multiple histone 
527 modifications (typical for both heterochromatin and euchromatin) in closely-related 528 phytopathogenic fungi. Nothing is known about how differences in the location of these 529 modifications influence pathogenesis. Comparative genomics has allowed analyses regarding the 530 evolution of genes (notably proteinaceous effectors), the location of effector genes in genomes, 531 or the diversity of effector repertoires in relation to host specialisation or fungal lifestyles. The 532 role of the epigenome is increasingly recognized in plant-pathogenic fungi as an important 533 regulator of genome structure (e.g. 70, 71, 80) and the expression of genes encoding effectors 534 (e.g. 32, 33, 35). The next step is to exploit comparative epigenomics to better understand the 535 role of the epigenomic landscape in adaptation to environmental changes, modulation of 536 interactions with the holobiont, host adaptation and specialization.

\section{Authors' contributions}

539 Conceived, initiated and coordinated the project: JLS, TR, EHS, IF. Designed the experiments: JLS, 540 EHS, IF. Performed the experiments: JLS. Analysis of the data: JLS, CC, EJG, NL. Interpretation of 541 the data: JLS, TR, EHS, IF. Writing of the draft: JLS. Editing of the draft: JLS, CC, TR, EHS, IF.

\section{Acknowledgments}

544 Authors wish to thank all members of the "Effectors and Pathogenesis of L. maculans" and 545 "Environmental Genomics" groups; M. Freitag and J. Grandaubert for fruitful discussions; R. 546 O'Connell for the English proofreading of this paper.

\section{Funding}


549 J. L. Soyer was funded by a "Contrat Jeune Scientifique" grant from INRA. The "Effectors and 550 Pathogenesis of L. maculans" group benefits from the support of Saclay Plant Sciences-SPS 551 (ANR-17-EUR-0007). Research on fungal histone modifications in the group of E.H. Stukenbrock 552 is funded by the Max Planck Society, Germany. The funders had no role in study design, data 553 collection and interpretation, or the decision to submit the work for publication.

554

555 Availability of data and material

556 Omic data are available under the GEO accession number GSE150127.

558 Ethics approval and consent to participate

559 Not applicable.

560

561 Consent for publication

562 Not applicable.

563

564 Conflict of interest

565 None to declare.

566

567 Competing interests

568 Authors declare no competing interest.

569

570 References 
571 1. Fisher MC, Hawkins NJ, Sanglard D, Gurr SJ. Worldwide emergence of resistance to antifungal 572 drugs challenges human health and food security. Science. 2018;360:739-42.

573 2. Rouxel T, Balesdent MH. Life, death and rebirth of avirulence effectors in a fungal pathogen of 574 Brassica crops, Leptosphaeria maculans. New Phytol. 2017;214:526-32.

575 3. Stukenbrock EH, Bataillon T, Dutheil JY, Hansen TT, Li R, Zala M, et al. The making of a new 576 pathogen: insights from comparative population genomics of the domesticated wheat pathogen 577 Mycosphaerella graminicola and its wild sister species. Genome Res. 2011;21:2157-66.

578 4. Grandaubert J, Lowe RG, Soyer JL, Schoch CL, Van de Wouw AP, Fudal I, et al. Transposable 579 element-assisted evolution and adaptation to host plant within the Leptosphaeria maculans580 Leptosphaeria biglobosa species complex of fungal pathogens. BMC Genomics. 2014;15:891.

581 5. Sánchez-Vallet A, Fouché S, Fudal I, Hartmann FE, Soyer JL, Tellier A, et al. The genome biology 582 of effector gene evolution in filamentous plant pathogens. Annu Rev Phytopathol. 2018;56:2158340.

584 6. Rouxel T, Grandaubert J, Hane JK, Hoede C, van de Wouw AP, Couloux A, et al. Effector 585 diversification within compartments of the Leptosphaeria maculans genome affected by Repeat586 Induced Point mutations. Nat Commun. 2011;2:202.

587 7. Ohm RA, Feau N, Henrissat B, Schoch CL, Horwitz BA, Barry KW, et al. Diverse lifestyles and 588 strategies of plant pathogenesis encoded in the genomes of eighteen Dothideomycetes fungi. 589 PLoS Pathog. 2012;8:e1003037.

590 8. Croll D, McDonald BA. The accessory genome as a cradle for adaptive evolution in pathogens. 591 PLoS Pathog. 2012;8:e1002608.

592 9. de Jonge R, Bolton MD, Kombrink A, van den Berg GC, Yadeta KA, Thomma BP. Extensive 
593 chromosomal reshuffling drives evolution of virulence in an asexual pathogen. Genome Res. $5942013 ; 23: 1271-82$.

595 10. Lo Presti L, Lanver D, Schweizer G, Tanaka S, Liang L, Tollot M, et al. Fungal effectors and 596 plant susceptibility. Annu Rev Plant Biol. 2015;66:513-45.

597 11. Collemare J, O'Connell R, Lebrun M-H. Nonproteinaceous effectors: the terra incognita of 598 plant-fungal interactions. New Phytol. 2019;223:590-6.

599 12. Rocafort M, Fudal I, Mesarich CH. Apoplastic effector proteins of plant-associated fungi and 600 oomycetes. Curr Opin Plant Biol. 2020;56:9-19.

601 13. Stergiopoulos I, De Kock MJ, Lindhout P, De Wit PJ. Allelic variation in the effector genes of 602 the tomato pathogen Cladosporium fulvum reveals different modes of adaptive evolution. Mol 603 Plant Microbe Interact. 2007;20:1271-83.

604 14. Chuma I, Isobe C, Hotta Y, Ibaragi K, Futamata N, Kusaba M, et al. Multiple translocation of 605 the AVR-Pita effector gene among chromosomes of the rice blast fungus Magnaporthe oryzae 606 and related species. PLoS Pathog. 2011;7:e1002147.

607 15. Jones JD, Dangl JL. The plant immune system. Nature. 2006;444:323-9.

608 16. Rouxel T, Balesdent MH. The stem canker (blackleg) fungus, Leptosphaeria maculans, enters 609 the genomic era. Mol Plant Pathol. 2005;6:225-41.

610 17. Gout L, Fudal I, Kuhn M-L, Blaise F, Eckert M, Cattolico L, et al. Lost in the middle of nowhere: 611 the AvrLm1 avirulence gene of the Dothideomycete Leptosphaeria maculans. Mol Microbiol. $612 \quad 2006 ; 60: 67-80$.

613 18. Fudal I, Ross S, Gout L, Blaise F, Kuhn ML, Eckert MR, et al. Heterochromatin-like regions as 614 ecological niches for avirulence genes in the Leptosphaeria maculans genome: map-based 
615 cloning of AvrLm6. Mol Plant Microbe Interact. 2007;20:459-70.

616 19. Parlange F, Daverdin G, Fudal I, Kuhn M-L, Balesdent M-H, Blaise F, et al. Leptosphaeria 617 maculans avirulence gene AvrLm4-7 confers a dual recognition specificity by the $R \operatorname{lm} 4$ and $R / m 7$ 618 resistance genes of oilseed rape, and circumvents RIm4-mediated recognition through a single 619 amino acid change. Mol Microbiol. 2009;71:851-63.

620 20. Balesdent M-H, Fudal I, Ollivier B, Bally P, Grandaubert J, Eber F, et al. The dispensable 621 chromosome of Leptosphaeria maculans shelters an effector gene conferring avirulence towards 622 Brassica rapa. New Phytol. 2013;198:887-898.

623 21. Van de Wouw AP, Lowe RG, Elliott CE, Dubois DJ, Howlett BJ. An avirulence gene, AvrLmJ1, 624 from the blackleg fungus, Leptosphaeria maculans, confers avirulence to Brassica juncea 625 cultivars. Mol Plant Pathol. 2014;15:523-30.

626 22. Ghanbarnia K, Fudal I, Larkan NJ, Links MG, Balesdent M-H, Profotova B, et al. Rapid 627 identification of the Leptosphaeria maculans Avirulence gene AvrLm2 using an intraspecific 628 comparative genomics approach. Mol Plant Pathol. 2015;16:699-709.

629 23. Ghanbarnia K, Ma L, Larkan NJ, Haddadi P, Fernando WGD, Borhan MH. Leptosphaeria 630 maculans AvrLm9: a new player in the game of hide and seek with AvrLm4-7. Mol Plant Pathol. $6312018 ; 19:$ 1754-64.

632 24. Plissonneau C, Daverdin G, Ollivier B, Blaise F, Degrave A, Fudal I, et al. A game of hide and 633 seek between avirulence genes AvrLm4-7 and AvrLm3 in Leptosphaeria maculans. New Phytol. 634 2016;209:1613-24.

635 25. Plissonneau C, Rouxel T, Chèvre A-M, Van De Wouw AP, Balesdent $M-H$. One gene-one name: 636 the AvrLmJ1 avirulence gene of Leptosphaeria maculans is AvrLm5. Mol Plant Pathol. 
638 26. Petit-Houdenot Y, Degrave A, Meyer M, Blaise F, Ollivier B, Marais C-L, et al. A two genes - for 639 - one gene interaction between Leptosphaeria maculans and Brassica napus. New Phytol. $640 \quad 2019 ; 223: 397-411$.

641 27. Gervais J, Plissonneau C, Linglin J, Meyer M, Labadie K, Cruaud C, et al. Different waves of 642 effector genes with contrasted genomic location are expressed by Leptosphaeria maculans 643 during cotyledon and stem colonization of oilseed rape. Mol Plant Pathol. 2017;18:1113-26.

644 28. Zhao Y, Garcia BA. Comprehensive catalog of currently documented histone modifications. 645 Cold Spring Harb Perspect Biol. 2015;7:a025064.

646 29. Gomes MV, Pelosi GG. Epigenetic vulnerability and the environmental influence on health.

647 Exp Biol Med (Maywood). 2013;238:859-65.

648 30. Zogli P, Libault M. Plant response to biotic stress: Is there a common epigenetic response 649 during plant-pathogenic and symbiotic interactions? Plant Sci. 2017;263:89-93.

650 31. Connolly LR, Smith KM, Freitag M. The Fusarium graminearum histone H3K27 651 methyltransferase KMT6 regulates development and expression of secondary metabolite gene 652 clusters. PLoS Genet. 2013;9:e1003916.

653 32. Chujo T, Scott B. Histone H3K9 and H3K27 methylation regulates fungal alkaloid biosynthesis 654 in a fungal endophyte-plant symbiosis: K9 and K27 methylation regulates symbiosis. Mol 655 Microbiol. 2014;92:413-34.

656 33. Soyer JL, El Ghalid M, Glaser N, Ollivier B, Linglin J, Grandaubert J, et al. Epigenetic control of 657 effector gene expression in the plant pathogenic fungus Leptosphaeria maculans. PLoS Genet. $658 \quad 2014 ; 10: e 1004227$. 
659 34. Soyer JL, Rouxel T, Fudal I. A. Chromatin-based control of effector gene expression in plant660 associated fungi. Curr Opin Plant Biol. 2015;26:51-6.

661 35. Soyer JL, Grandaubert J, Haueisen J, Schotanus K, Stukenbrock EH. In planta chromatin 662 immunoprecipitation in Zymoseptoria tritici reveals chromatin-based regulation of putative 663 effector gene expression. 2019. Preprint at bioRxiv. https://doi.org/10.1101/544627

664 36. Daverdin G, Rouxel T, Gout L, Aubertot JN, Fudal I, Meyer M, et al. Genome structure and 665 reproductive behaviour influence the evolutionary potential of a fungal phytopathogen. PLoS 666 Pathog. $2012 ; 8: 1003020$.

667 37. Dutreux F, Da Silva C, d'Agata L, Couloux A, Gay EJ, Istace B, et al. De novo assembly and 668 annotation of three Leptosphaeria genomes using Oxford Nanopore MinION sequencing. Sci 669 Data; 2018;5:180235.

670 38. Petrie GA. Variability in Leptosphaeria maculans (Desm.) Ces. et De Not., the cause of 671 blackleg of rape. 1969. PhD thesis, University of Saskatchewan (215 pp.).

672 39. Zhang Z, Wen J, Li J, Ma X, Yu Y, Tan X, et al. The evolution of genomic and epigenomic 673 features in two Pleurotus fungi. Sci Rep. 2018;8:8313.

674 40. Feurtey A, Lorrain C, Croll D, Eschenbrenner C, Freitag M, Habig M, et al. Genome 675 compartmentalization predates species divergence in the plant pathogen genus Zymoseptoria. 676 2019. Preprint at bioRXiv. https://doi.org/10.1101/864561

677 41. Ansan-Melayah D, Balesdent M-H, Buee M, Rouxel T. Genetic characterization of AvrLm1, the 678 first avirulence gene of Leptosphaeria maculans. Phytopathology. 1995;85:1525-9.

679 42. Soyer JL, Möller M, Schotanus K, Connolly LR, Galazka JM, Freitag M, et al. Chromatin 680 analyses of Zymoseptoria tritici: Methods for chromatin immunoprecipitation followed by high- 
681 throughput sequencing (ChIP-seq). Fungal Genet Biol. 2015;79:63-70.

682 43. Schotanus K, Soyer JL, Connolly LR, Grandaubert J, Happel P, Smith KM, et al. Histone 683 modifications rather than the novel regional centromeres of Zymoseptoria tritici distinguish core 684 and accessory chromosomes. Epigenetics Chromatin. 2015;8:41.

685 44. Langmead B, Salzberg SL. Fast gapped-read alignment with Bowtie 2. Nat Methods. $6862012 ; 9: 357-9$.

687 45. Song Q, Smith AD. Identifying dispersed epigenomic domains from ChIP-Seq data. 688 Bioinformatics. 2011;27:870-1.

689 46. Thorvaldsdóttir H, Robinson JT, Mesirov JP. Integrative Genomics Viewer (IGV): high690 performance genomics data visualization and exploration. Brief Bioinform. 2013;14:178-92.

691 47. Bolger AM, Lohse M, Usadel B. Trimmomatic: a flexible trimmer for Illumina sequence data. 692 Bioinformatics. 2014;30:2114-20.

693 48. Dobin A, Gingeras TR. Mapping RNA-seq reads with STAR. Current Protoc Bioinformatics. $6942015 ; 3,51: 11.14 .1-11.14 .19$.

695 49. Robinson MD, McCarthy DJ, Smyth GK. edgeR: a Bioconductor package for differential 696 expression analysis of digital gene expression data. Bioinformatics. 2010;26:139-40.

697 50. Emms DM, Kelly S. OrthoFinder: solving fundamental biases in whole genome comparisons 698 dramatically improves orthogroup inference accuracy. Genome Biol. 2015;16:157.

699 51. Petersen TN, Brunak S, von Heijne G, Nielsen H. SignalP 4.0: discriminating signal peptides 700 from transmembrane regions. Nat Methods. 2011;8:785-6.

701 52. Almagro Armenteros JJ, Salvatore M, Emanuelsson O, Winther O, von Heijne G, Elofsson A, et 702 al. Detecting sequence signals in targeting peptides using deep learning. Life Sci Alliance. 
704 53. Sperschneider J, Gardiner DM, Dodds PN, Tini F, Covarelli L, Singh KB, et al. EffectorP: 705 predicting fungal effector proteins from secretomes using machine learning. New phytol. $706 \quad 2016 ; 210: 743-61$.

707 54. Shannon P, Markiel A, Ozier O, Baliga NS, Wang JT, Ramage D, et al. Cytoscape: a software 708 environment for integrated models of biomolecular interaction networks. Genome Res. 709 2003;13:2498-504.

710 55. Leclair S, Ansan-Melayah D, Rouxel T, Balesdent M-H. Meiotic behaviour of the 711 minichromosome in the phytopathogenic ascomycete Leptosphaeria maculans. Curr Genet. 712 1996;30:541-8.

713 56. Darma R, Lutz A, Elliott CE, Idnurm A. Identification of a gene cluster for the synthesis of the 714 plant hormone abscisic acid in the plant pathogen Leptosphaeria maculans. Fungal Genet Biol. 715 2019;130:62-71.

716 57. Elliott CE, Callahan DL, Schwenk D, Nett M, Hoffmeister D, Howlett BJ. A gene cluster 717 responsible for biosynthesis of phomenoic acid in the plant pathogenic fungus, Leptosphaeria 718 maculans. Fungal Genet Biol. 2013;53:50-8.

719 58. Gardiner DM, Cozijnsen AJ, Wilson LM, Pedras MS, Howlett BJ. The sirodesmin biosynthetic 720 gene cluster of the plant pathogenic fungus Leptosphaeria maculans. Mol Microbiol. $721 \quad 2004 ; 53: 1307-18$.

722 59. Elliott CE, Gardiner DM, Thomas G, Cozijnsen A, Van De Wouw A, Howlett BJ. Production of 723 the toxin sirodesmin PL by Leptosphaeria maculans during infection of Brassica napus. Mol Plant 724 Pathol. 2007;8:791-802. 
725 60. Smith KM, Kothe GO, Matsen CB, Khlafallah TK, Adhvaryu KK, Hemphill M, et al. The fungus 726 Neurospora crassa displays telomeric silencing mediated by multiple sirtuins and by methylation 727 of histone H3 lysine 9. Epigenetics Chromatin. 2008;1:5.

728 61. Jamieson K, Rountree MR, Lewis ZA, Stajich JE, Selker EU. Regional control of histone H3 729 lysine 27 methylation in Neurospora. Proc Natl Acad Sci U S A. 2013;110:6027-32.

730 62. Wiemann P, Sieber CM, von Bargen KW, Studt L, Niehaus EM, Espino JJ, et al. Deciphering the 731 cryptic genome: genome-wide analyses of the rice pathogen Fusarium fujikuroi reveal complex 732 regulation of secondary metabolism and novel metabolites. PLoS Pathog. 2013;9:e1003475.

733 63. Gacek-Matthews A, Berger H, Sasaki T, Wittstein K, Gruber C, Lewis ZA, et al. KdmB, a 734 Jumonji Histone $\mathrm{H} 3$ demethylase, regulates genome-wide $\mathrm{H} 3 \mathrm{~K} 4$ trimethylation and is required 735 for normal induction of secondary metabolism in Aspergillus nidulans. PLoS Genet. $736 \quad$ 2016;12:e1006222.

737 64. Freitag M. Histone methylation by SET domain proteins in Fungi. Annu Rev Microbiol. $738 \quad 2017 ; 71: 413-39$.

739 65. Jamieson K, McNaught KJ, Ormsby T, Leggett NA, Honda S, Selker EU. Telomere repeats 740 induce domains of H3K27 methylation in Neurospora. Elife. 2018;7:e31216.

741 66. Fokkens L, Shahi S, Connolly LR, Stam R, Schmidt SM, Smith KM, et al. The multi-speed 742 genome of Fusarium oxysporum reveals association of histone modifications with sequence 743 divergence and footprints of past horizontal chromosome transfer events. 2018. Preprint at 744 bioRXiv. https://doi.org/10.1101/465070

745 67. Bernstein BE, Mikkelsen TS, Xie X, Kamal M, Huebert DJ, Cuff J, et al. A bivalent chromatin 746 structure marks key developmental genes in embryonic stem cells. Cell. 2006;125:315-26. 
747 68. Harikumar A, Meshorer E. Chromatin remodeling and bivalent histone modifications in 748 embryonic stem cells. EMBO Rep. 2015;16:1609-19.

749 69. Grewal SI, Jia S. Heterochromatin revisited. Nat Rev Genet. 2007;8:35-46.

750 70. Möller M, Schotanus K, Soyer JL, Haueisen J, Happ K, Stralucke M, et al. Destabilization of 751 chromosome structure by histone H3 lysine 27 methylation. PLoS Genet. 2019;15:e1008093.

752 71. Basenko EY, Sasaki T, Ji L, Prybol CJ, Burckhardt RM, Schmitz RJ, et al. Genome-wide 753 redistribution of $\mathrm{H} 3 \mathrm{~K} 27 \mathrm{me} 3$ is linked to genotoxic stress and defective growth. Proc Natl Acad Sci 754 U S A. 2015;112:E6339-48.

755 72. Tan KC, Oliver RP. Regulation of proteinaceous effector expression in phytopathogenic fungi. 756 PLoS Pathog. 2017;13:e1006241.

757 73. Ma LJ, van der Does HC, Borkovich KA, Coleman JJ, Daboussi MJ, Di Pietro A, et al. 758 Comparative genomics reveals mobile pathogenicity chromosomes in Fusarium. Nature. $7592010 ; 464: 367-73$.

760 74. Faino L, Seidl MF, Shi-Kunne X, Pauper M, van den Berg GC, Wittenberg AH, et al. 761 Transposons passively and actively contribute to evolution of the two-speed genome of a fungal 762 pathogen. Genome Res. 2016;26:1091-1100.

763 75. Vlaardingerbroek I, Beerens B, Schmidt SM, Cornelissen BJ, Rep M. Dispensable 764 chromosomes in Fusarium oxysporum f. sp. Iycopersici. Mol Plant Pathol. 2016;17:1455-66.

765 76. Dallery JF, Lapalu N, Zampounis A, Pigné S, Luyten I, Amselem J, et al. Gapless genome 766 assembly of Colletotrichum higginsianum reveals chromosome structure and association of 767 transposable elements with secondary metabolite gene clusters. BMC Genomics. 2017;18:667. 768 77. Krishnan P, Meile L, Plissonneau C, Ma X, Hartmann FE, Croll D, et al. Transposable element 
769 insertions shape gene regulation and melanin production in a fungal pathogen of wheat. BMC 770 Biol. 2018;16:78.

771 78. Haueisen J, Möller M, Eschenbrenner CJ, Grandaubert J, Seybold H, Adamiak H, et al. Highly

772 flexible infection programs in a specialized wheat pathogen. Ecol Evol. 2018;9:275-94.

773 79. Mikkelsen TS, Xu Z, Zhang X, Wang L, Gimble JM, Lander ES, et al. Comparative epigenomic 774 analysis of murine and human adipogenesis. Cell. 2010;143:156-69.

775 80. Möller M, Habig M, Lorrain C, Feurtey A, Haueisen J, Fagundes WC, et al. Recent loss of the 776 Dim2 cytosine DNA methyltransferase impacts mutation rate and evolution in a fungal plant 777 pathogen. 2020. Preprint at bioRXiv. https://doi.org/10.1101/2020.03.27.012203

778

779 Figure legends

780 Figure 1. Size of the histone domains in the genomes of Leptosphaeria maculans 'brassicae' 781 and L. maculans 'Iepidii'. Log2 of the size of the domains was estimated based on the 782 coordinates of the location of the domains, identified using RSEG (45). Blue: H3K4me2; Purple: 783 H3K9me3; Orange: H3K27me3; Lmb: L. maculans 'brassicae'; Lml: L. maculans 'lepidii'.

784

785 Figure 2. Genome of Leptosphaeria maculans 'brassicae' harbors large TE-rich, H3K9me3786 domains compared to Leptosphaeria maculans 'lepidii'. Example of SuperContig 2 of Lmb and 787 Scaffold 1 of Lml. ChIP-seq was performed with antibodies targeting H3K4me2 (cyan), H3K9me3 788 (purple) or $\mathrm{H} 3 \mathrm{~K} 27 \mathrm{me} 3$ (orange); rectangles indicate location of significantly enriched domains 789 identified using RSEG (45). Blue: location of CDS; red: location of transposable elements; Lmb: $L$. 790 maculans 'brassicae'; Lml: L. maculans 'lepidii'. Genes encoding proteinaceous effectors are 
791 indicated with a red square.

792

793 Figure 3. Coverage of different genome features in the genomes of L. maculans 'brassicae' and

794 L. maculans 'lepidii'. A. SuperContig 2 of Lmb; B. SC 19, i.e. dispensable chromosome, of Lmb; C.

795 SC 1 of $\mathrm{Lml}$. SC2 of $\mathrm{Lmb}$ and SC1 of $\mathrm{Lml}$ are syntenic (4). Coverage of the genome features and

796 histone modification domains, as identified in axenic cultures, were analysed in $10 \mathrm{~kb}$ sliding

797 windows in the genomes of Lmb and Lml. Lmb, L. maculans 'brassicae'; Lml, L. maculans 'lepidii';

798 CDS, Coding Sequences; TE, Transposable Elements; SC, Super Contig.

799

800 Figure 4. Number of genes associated with histone modifications in Leptosphaeria maculans

801 'brassicae' and L. maculans 'lepidii'. A. L. maculans 'brassicae', Lmb and B. L. maculans 'lepidii',

$802 \mathrm{Lml}$. Locations of histone modifications in the genomes of $\mathrm{Lmb}$ and $\mathrm{Lml}$ were identified using

803 RSEG (45). Blue: H3K4me2; Purple: H3K9me3; Orange: H3K27me3. Genes were considered as

804 associated with any of the histone modifications when at least one bp of the gene was found

805 within the borders of the domain.

806

807 Figure 5. Genes associated with heterochromatin are less expressed than genes associated

808 with euchromatin in L. maculans 'brassicae' and L. maculans 'lepidii'. A. L. maculans

809 'brassicae'; B. L. maculans 'lepidii'. Location of histone modifications in the genomes of Lmb and

810 Lml were identified using RSEG (45), during axenic culture. RNA-seq was performed from Lmb or

811 Lml grown one week in FRIES media. Blue: H3K4me2; Purple: H3K9me3; Orange: H3K27me3. 
Table 3. Number of proteinaceous effector genes located in different genomic compartments in $L$. maculans 'brassicae' and $L$. maculans 'lepidii'

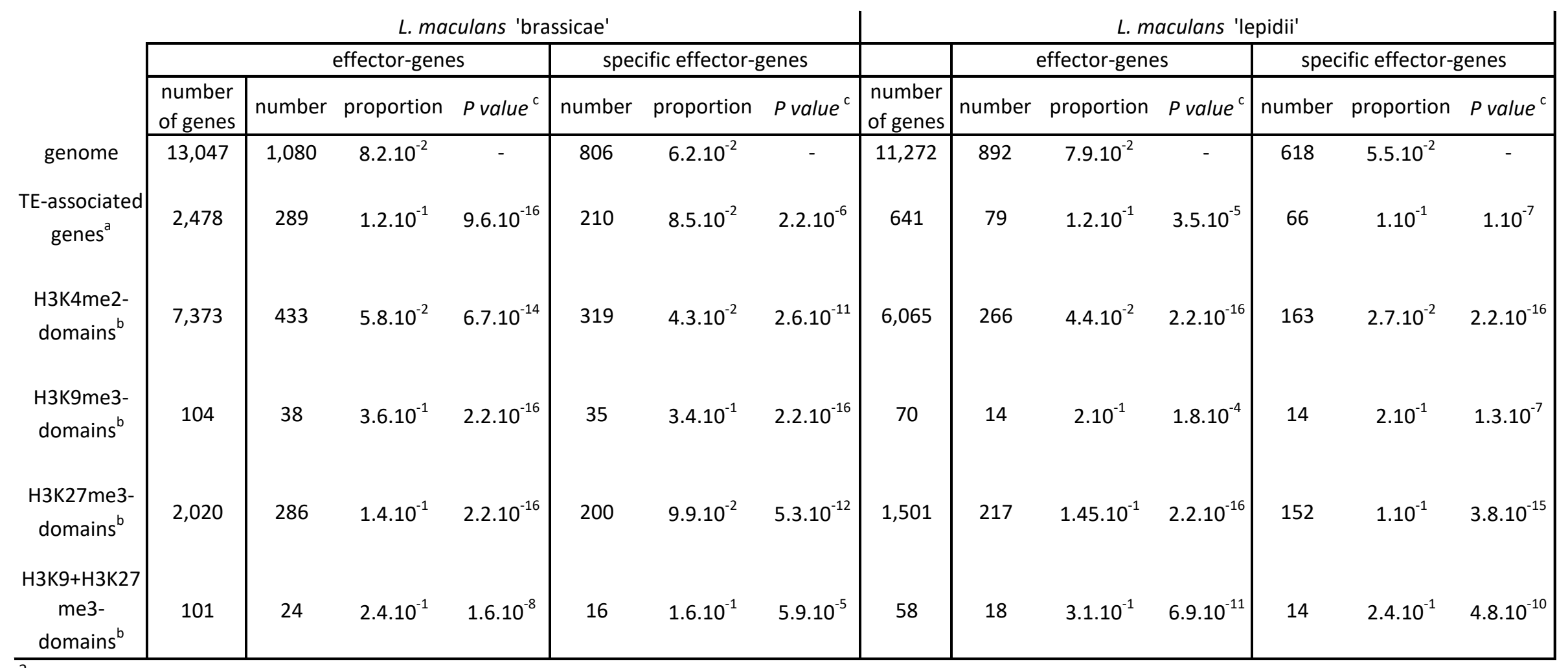

${ }^{\mathrm{a}}$ Genes located up to $2 \mathrm{~kb}$ upstream or downstream of a transposable element sequence;

${ }^{\mathrm{b}}$ Genes located in a H3K4me2-, H3K9me3-, H3K27me3- or H3K9me3/H3K27me3-domain in vitro ;

${ }^{\mathrm{C}} \mathrm{A} \mathrm{X}^{2}$ test was applied to compare proportion of effector genes in the genome and in the genomic compartment analysed. 


\section{Additional figure legends}

814 Additional figure 1. Correlation analysis of the location of genes, transposable elements and 815 domains enriched for H3K4me2, H3K9me3 and H3K27me3 in the genomes of A. Leptosphaeria 816 maculans 'brassicae'; B. Leptosphaeria maculans 'lepidii'. Regions significantly enriched for any

817 of the three histone modifications analysed were identified through ChIP-seq performed during 818 axenic culture; correlation analyses were performed using a Kendall's $\mathcal{T}$ (see Materials and

819 Methods). K4: H3K4me2; K9: H3K9me3; K27: H3K27me3; CDS: Coding sequences; TE: 820 Transposable Elements.

822 Additional table legends

823 Additional table 1. Statistics of ChIP-seq and RNA-seq datasets and alignments. Table shows 824 the number of reads used for the alignment, the number of reads mapping only at one location, 825 the number of reads aligned more than once and the unmapped reads against the genome of $L$. 826 maculans 'brassicae' (Lmb; 37) or L. maculans 'lepidii' (Lml; 4).

828 Additional table 2. Correlation analysis between the different ChIP experiments generated 829 with antibodies targeting histone modifications H3K4me2, H3K9me3 and H3K27me3 in $L$. 830 maculans 'brassicae', during in vitro growth. Correlation analyses were performed to analyse 831 location of the significantly enriched domains, identified using RSEG (45), from the three 832 different biological replicates generated and analyzed separately. A Kendall's $\mathcal{T}$ correlation test 833 was performed using $\mathrm{R}$. 
835 Additional table 3. Correlation analysis between the different ChIP experiments generated 836 with antibodies targeting histone modifications H3K4me2, H3K9me3 and H3K27me3 in L. 837 maculans 'lepidii', during in vitro growth.

838 Correlation analyses were performed to analyse location of the significantly enriched domains, 839 identified using RSEG (45), from the three different biological replicates generated and analyzed 840 separately. A Kendall's $\mathcal{T}$ correlation test was performed using R.

842 Additional table 4. Coverage of histone modifications H3K4me2, H3K9me3 and H3K27me3 in 843 the genome of Leptosphaeria maculans 'brassicae'.

$844{ }^{a}$ Genome as published in Dutreux et al. (37)

845 bLocation of H3K4me2, H3K9me3 and H3K27me3 was determined through ChIP-seq analysis, in 846 vitro, and regions significantly enriched for any of the modifications was identified using RSEG 847 (45).

848

849 Additional table 5. Coverage of histone modifications H3K4me2, H3K9me3 and H3K27me3 in 850 the genome of $L$. maculans 'lepidii'.

851 aGenome as published in Grandaubert et al. (4)

852 bLocation of H3K4me2, H3K9me3 and H3K27me3 was determined through ChIP-seq analysis, in 853 vitro, and regions significantly enriched for any of the modifications was identified using RSEG 854 (45). 
858 culture of $L$. maculans 'brassicae'. GO annotation of the $L m b$ genes were retrieved from Dutreux

859 et al. (37). Analysis of $\mathrm{GO}$ enrichment among the genes associated with H3K4me2 during axenic

860 culture of Lmb was performed using Cytoscape (Shannon et al., 2003).

862 Additional table 7. GO categories enriched in genes associated with H3K4me2 during axenic 863 culture of L. maculans 'lepidii'. GO annotation of the $\mathrm{Lml}$ genes were retrieved from 864 Grandaubert et al. (4). Analysis of GO enrichment among the genes associated with H3K4me2 865 during axenic culture of $\mathrm{Lml}$ was performed using Cytoscape (54).

866

Additional table 8. GO categories enriched in genes associated with H3K27me3 during axenic culture of L. maculans 'brassicae'. GO annotation of the Lmb genes were retrieved from Dutreux et al. (37). Analysis of $\mathrm{GO}$ enrichment among the genes associated with $\mathrm{H} 3 \mathrm{~K} 27 \mathrm{me} 3$ during axenic

870 culture of Lmb was performed using Cytoscape (54).

871

872 Additional table 9. GO categories enriched in genes associated with H3K27me3 during axenic 873 culture of L. maculans 'lepidii'. GO annotation of the $\mathrm{Lml}$ genes were retrieved from 874 Grandaubert et al. (4). Analysis of GO enrichment among the genes associated with H3K27me3 875 during axenic culture of $\mathrm{Lml}$ was performed using Cytoscape (54).

876

877 Additional table 10. Location of (putative) metabolic effector in L. maculans 'brassicae' and L. 878 maculans 'lepidii' genomic compartments. 
$879{ }^{\mathrm{a}}$ Genes located up to $2 \mathrm{~kb}$ upstream or downstream of a transposable element sequence;

$880{ }^{\mathrm{b}}$ Genes with RPKM $\geq 2$;

$881{ }^{c}$ Genes located in a H3K4me2-, H3K9me3-, H3K27me3- or H3K4me2/H3K27me3-domain in vitro;

882 K4: H3K4me2; K9: H3K9me3; K27:H3K27me3.

883 


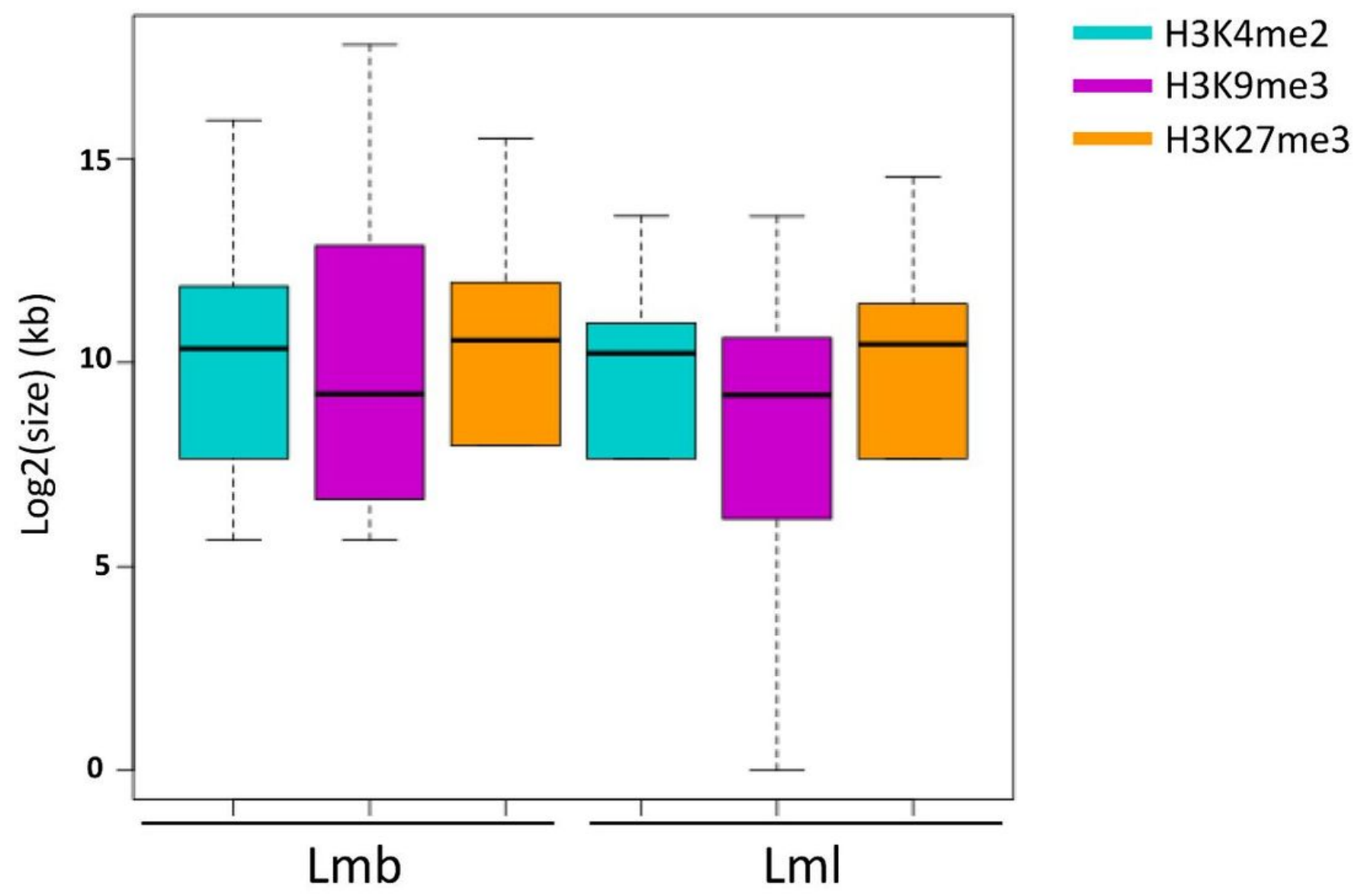

Figure 1

Size of the histone domains in the genomes of Leptosphaeria maculans 'brassicae' and L. maculans 'lepidii'. Log2 of the size of the domains was estimated based on the coordinates of the location of the domains, identified using RSEG (45). Blue: H3K4me2; Purple: H3K9me3; Orange: H3K27me3; Lmb: L. maculans 'brassicae'; Lml: L. maculans 'lepidii'. 


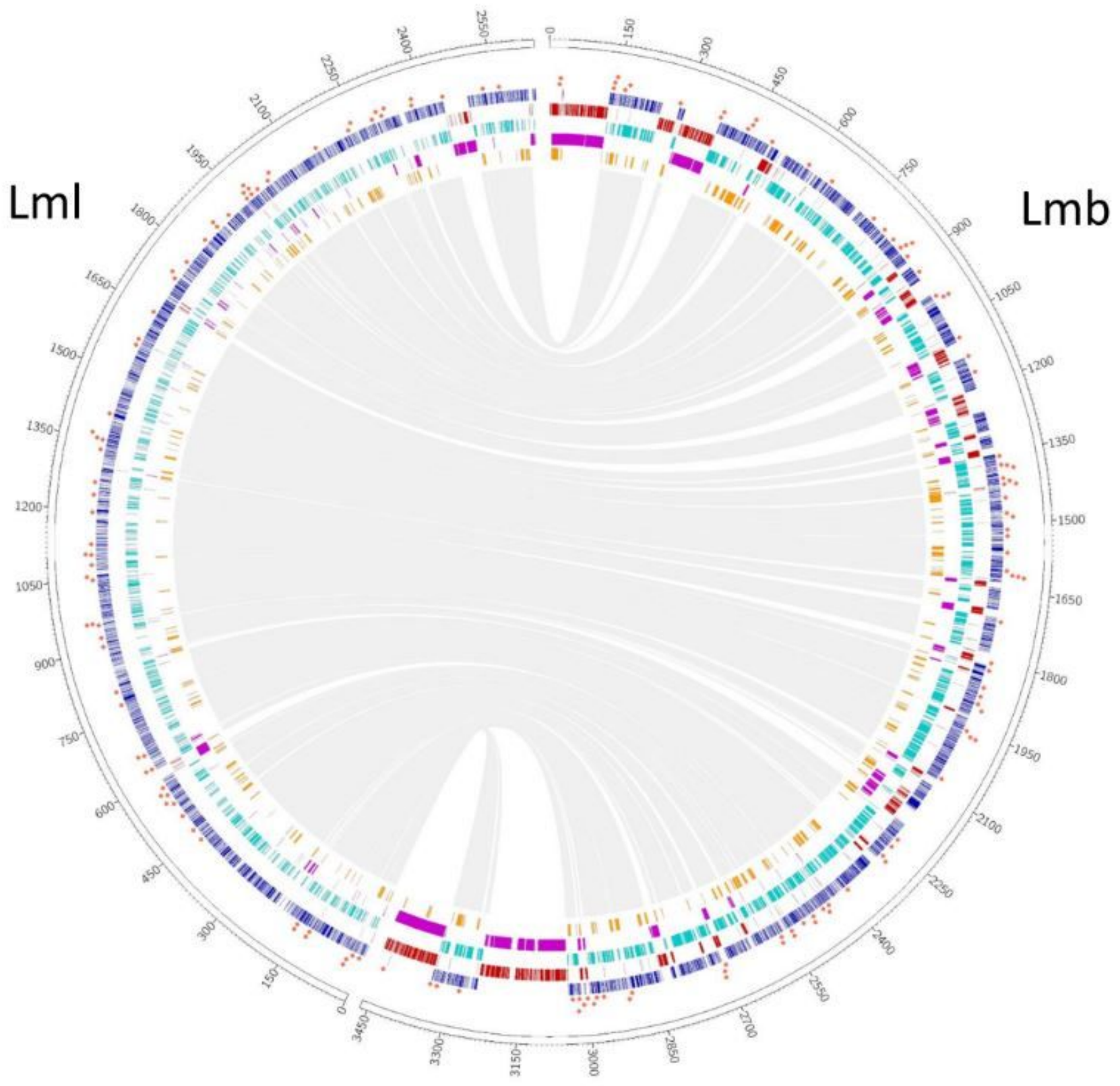

Figure 2

Genome of Leptosphaeria maculans 'brassicae' harbors large TE-rich, H3K9me3-domains compared to Leptosphaeria maculans 'lepidii'. Example of SuperContig 2 of Lmb and Scaffold 1 of Lml. ChIP-seq was performed with antibodies targeting H3K4me2 (cyan), H3K9me3 (purple) or H3K27me3 (orange); rectangles indicate location of significantly enriched domains identified using RSEG (45). Blue: location 
of CDS; red: location of transposable elements; Lmb: L. maculans 'brassicae'; Lml: L. maculans 'lepidii'. Genes encoding proteinaceous effectors are indicated with a red square.
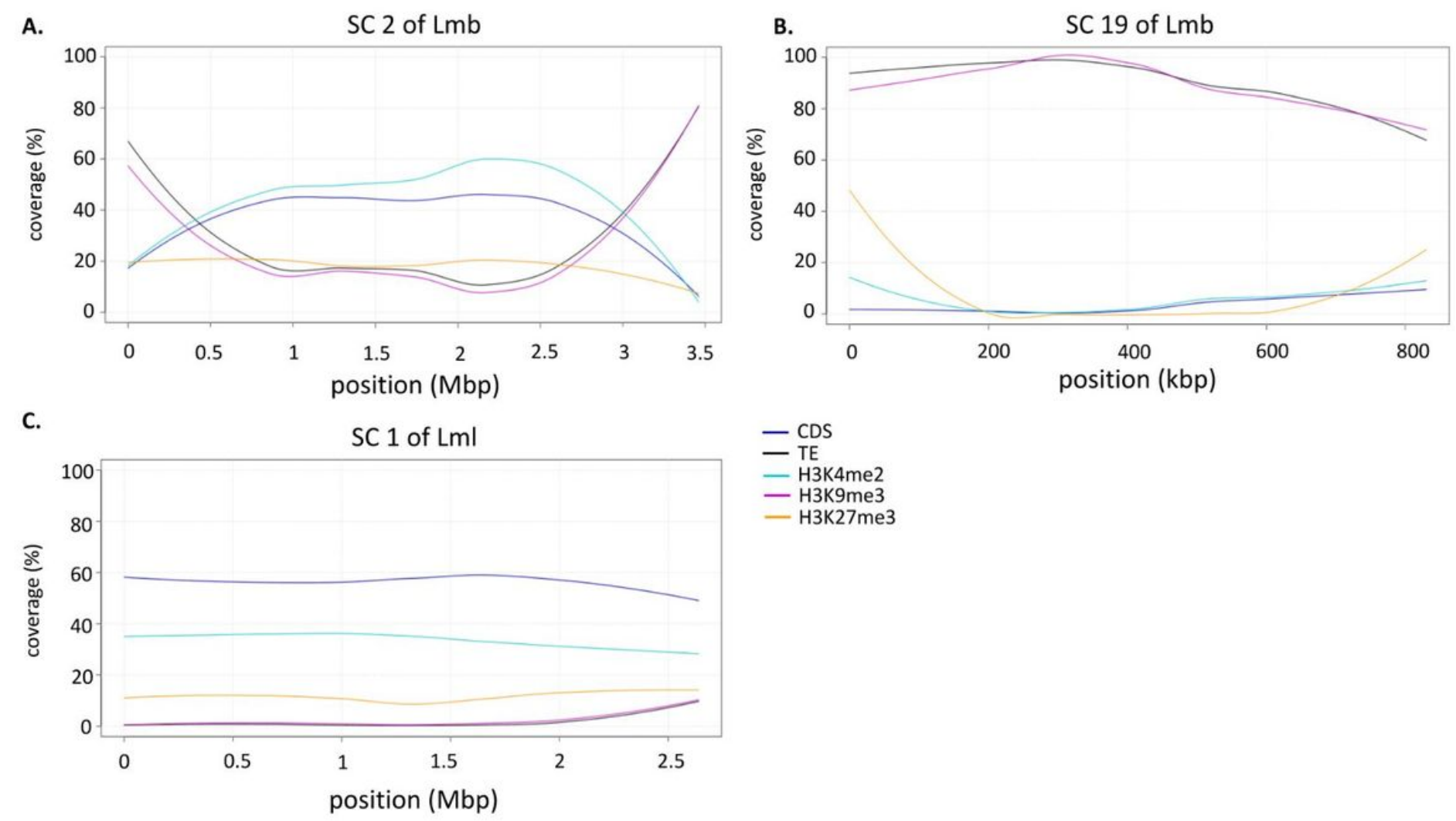

\section{Figure 3}

Coverage of different genome features in the genomes of L. maculans 'brassicae' and L. maculans 'lepidii'. A. SuperContig 2 of Lmb; B. SC 19, i.e. dispensable chromosome, of Lmb; C. SC 1 of Lml. SC2 of $\mathrm{Lmb}$ and SC1 of $\mathrm{Lml}$ are syntenic (4). Coverage of the genome features and histone modification domains, as identified in axenic cultures, were analysed in $10 \mathrm{~kb}$ sliding windows in the genomes of Lmb and Lml. Lmb, L. maculans 'brassicae'; Lml, L. maculans 'lepidii'; CDS, Coding Sequences; TE, Transposable Elements; SC, Super Contig. 
A.

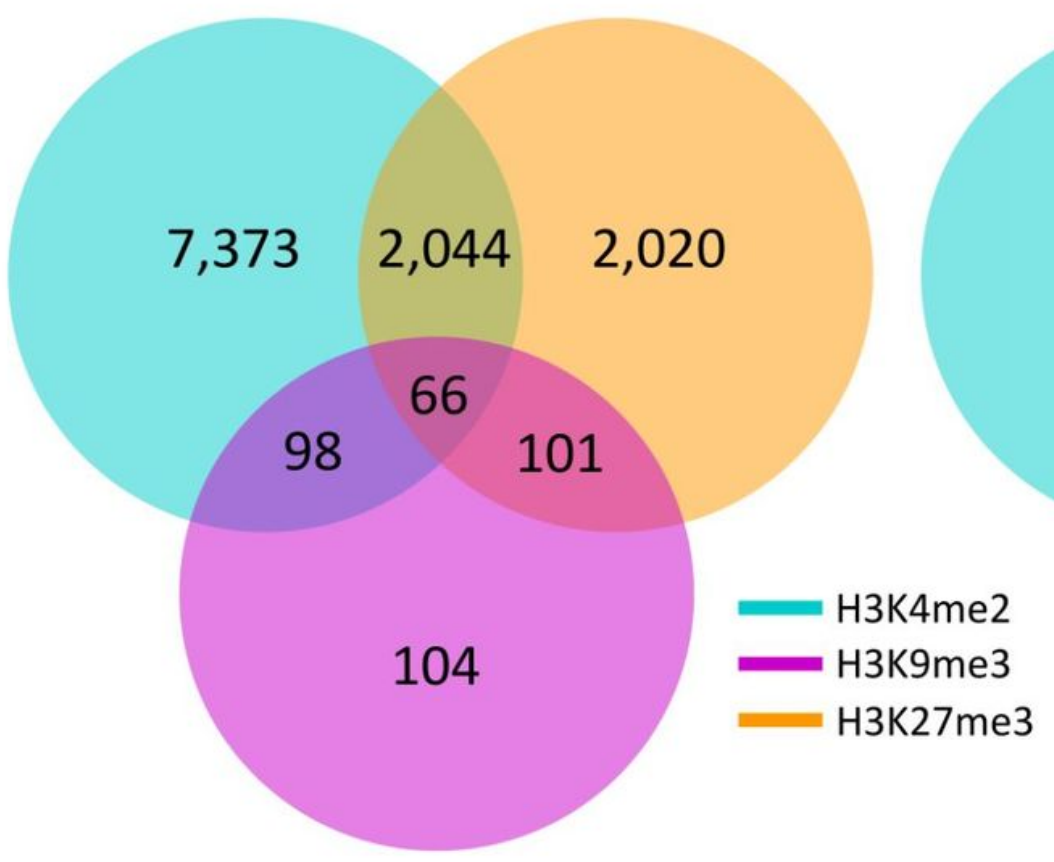

B.
L. maculans 'lepidii'

L. maculans 'lepidii'

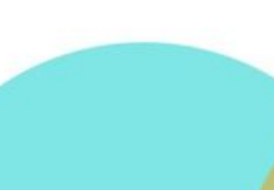

$6,065 \quad 361 \quad 1,501$

33

28

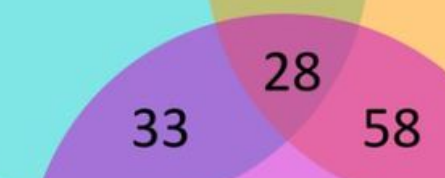

70

\section{Figure 4}

Number of genes associated with histone modifications in Leptosphaeria maculans 'brassicae' and L. maculans 'lepidii'. A. L. maculans 'brassicae', Lmb and B. L. maculans 'lepidii', Lml. Locations of histone modifications in the genomes of Lmb and Lml were identified using RSEG (45). Blue: H3K4me2; Purple: H3K9me3; Orange: H3K27me3. Genes were considered as associated with any of the histone modifications when at least one bp of the gene was found within the borders of the domain. 

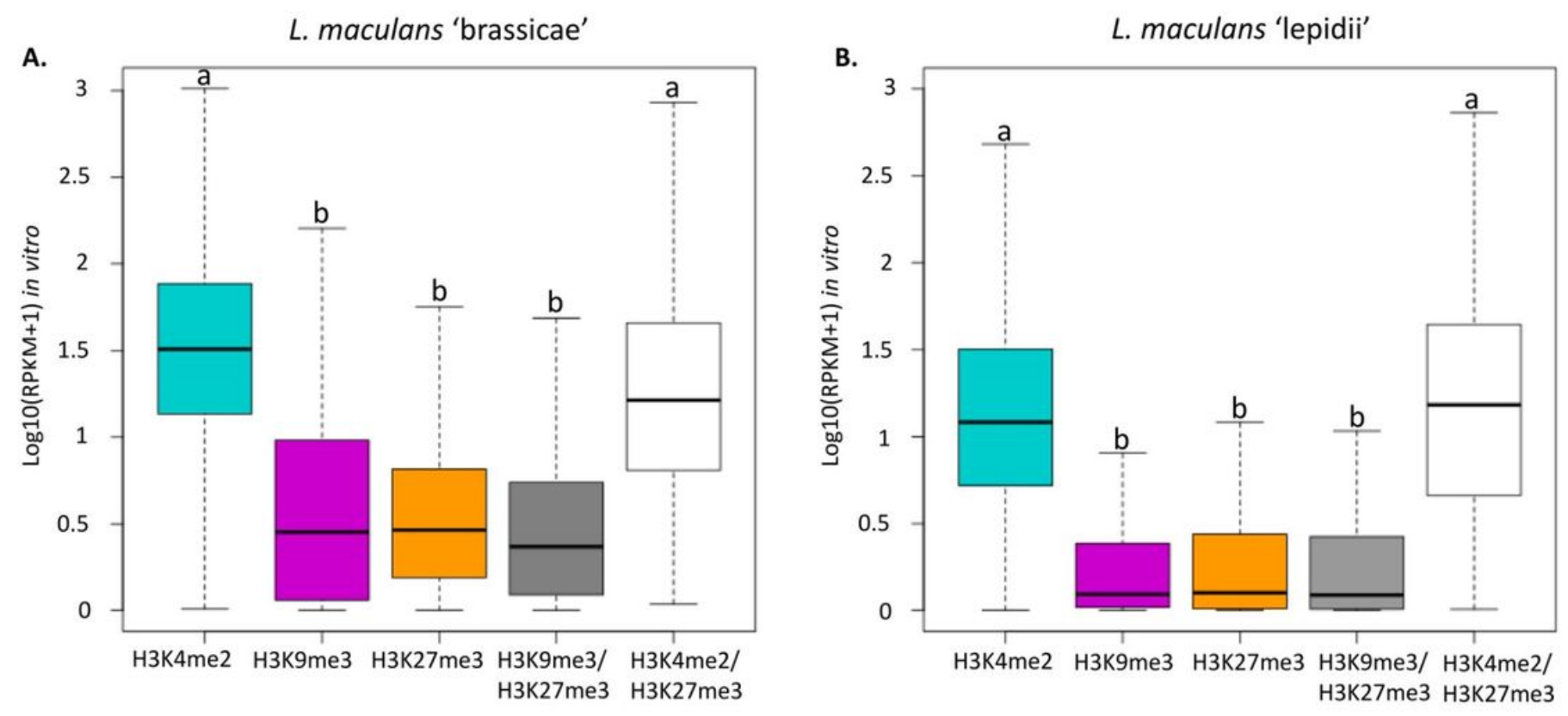

Figure 5

Genes associated with heterochromatin are less expressed than genes associated with euchromatin in L. maculans 'brassicae' and L. maculans 'lepidii'. A. L. maculans 'brassicae'; B. L. maculans 'lepidii'. Location of histone modifications in the genomes of Lmb and Lml were identified using RSEG (45), during axenic culture. RNA-seq was performed from Lmb or Lml grown one week in FRIES media. Blue: H3K4me2; Purple: H3K9me3; Orange: H3K27me3.

\section{Supplementary Files}

This is a list of supplementary files associated with this preprint. Click to download.

- Additionaltable10.pdf

- Additionaltable9.pdf

- Additionaltable8.pdf

- Additionaltable7.pdf

- Additionaltable6.pdf

- Additionaltable5.pdf

- Additionaltable4.pdf

- Additionaltable3.pdf

- Additionaltable2.pdf

- Additionaltable1.pdf 
- FigureS1.tiff 